TRANSACTIONS OF THE

AMERICAN MATHEMATICAL SOCIETY

Volume 362, Number 4, April 2010, Pages 2053-2087

S 0002-9947(09)05059-4

Article electronically published on November 18, 2009

\title{
CHRISTOFFEL FUNCTIONS ON CURVES AND DOMAINS
}

\author{
VILMOS TOTIK
}

\begin{abstract}
Asymptotics for Christoffel functions are established for measures supported on unions of smooth Jordan curves and for area-like measures on unions of smooth Jordan domains. For example, in the former case $n$ times the $n$-th Christoffel function tends to the Radon-Nikodym derivative of the measure with respect to the equilibrium distribution of the support of the measure.
\end{abstract}

\section{Contents}

1. Introduction and results

2. A few elementary facts about lemniscates and their domains

3. Equilibrium measures and Green's functions

4. Fast decreasing polynomials

5. Proof of Theorems 1.3 and 1.4

5.1. Proof of Theorem 1.3 for the unit disk and identically 1 weight 2067

5.2. Proof of Theorem 1.3 for lemniscate domains and for positive weights 2068

5.3. Proof of Theorem 1.3 in its full generality

5.4. Proof of Theorem 1.4

6. Proof of Theorems 1.1 and $1.2 \quad 2076$

6.1. Lower estimate for arc length measure 2076

6.2. Proof of Theorem [1.2, the lower estimate for all measures 2080

6.3. Proof of Theorem 1.2, upper estimate 2082

6.4. Proof of Theorem 1.1 2083

7. Markov and Bernstein type inequalities 2084

References $\quad 2085$

\section{INTRODUCTION AND RESUltS}

Let $\mu$ be a finite Borel measure on the plane such that its support is compact and consists of infinitely many points. The Christoffel functions associated with $\mu$ are defined as

$$
\lambda_{n}(\mu, z)=\inf _{P_{n}(z)=1} \int\left|P_{n}\right|^{2} d \mu,
$$

Received by the editors April 7, 2008.

2000 Mathematics Subject Classification. Primary 26C05, 31A99, 41A10.

The author was supported by NSF DMS 0700471.

(C)2009 American Mathematical Society Reverts to public domain 28 years from publication 
where the infimum is taken for all polynomials of degree at most $n$ that take the value 1 at $z$. If $p_{k}(z)=p_{k}(\mu, z)$ denotes the orthonormal polynomials with respect to $\mu$, i.e.

$$
\int p_{n} \overline{p_{m}} d \mu=\delta_{n, m}
$$

then $\lambda_{n}$ can be expressed as

$$
\lambda_{n}^{-1}(\mu, z)=\sum_{k=0}^{n}\left|p_{k}(z)\right|^{2} .
$$

In other words, $\lambda^{-1}(z)$ is the diagonal of the reproducing kernel

$$
K_{n}(z, w)=\sum_{k=0}^{n} p_{k}(z) \overline{p_{k}(w)}
$$

which makes it an essential tool in many problems. As early as 1915 G. Szegö proved that if $d \mu(t)=\mu^{\prime}(t) d t$ is an absolutely continuous measure on the unit circle (identified with $[-\pi, \pi]$ ), then

$$
\lim _{n \rightarrow \infty} \lambda_{n}(z)=\left(1-|z|^{2}\right) \exp \left(\frac{1}{2 \pi} \int_{-\pi}^{\pi} \frac{e^{i t}-z}{e^{i t}+z} \log \mu^{\prime}(t) d t\right), \quad|z|<1,
$$

provided $\log \mu^{\prime}(t)$ ) is integrable (otherwise the limit on the left is 0 ). This result was later generalized in various directions; see e.g. [13, [14], 8]. Szegö ([35, Th. I', p. 461]) also proved that on the unit circle

$$
\lim _{n \rightarrow \infty} n \lambda_{n}\left(\mu, e^{i \theta}\right)=2 \pi \mu^{\prime}(\theta)
$$

under the condition that $\mu$ is absolutely continuous and $\mu^{\prime}>0$ is twice continuously differentiable. The almost everywhere result came much later, only in 1991 was it proved in 18 that (1.1) is true almost everywhere provided $\log \mu^{\prime}$ is integrable.

In past literature much work has been devoted to Christoffel functions, e.g. the $H^{p}$ theory emerged from Szego''s theorem, the density of states in statistical mechanical models of quantum physics is given by the reciprocal of the Christoffel function associated with the spectral measure (see e.g. [23]), and the recent breakthrough [16] by Lubinsky in universality connected with random matrices has also been based on them (cf. also [4, 38, and particularly [31, where the importance of Christoffel functions regarding off diagonal behavior of the reproducing kernel was emphasized). See [5], 8, 29], and particularly [21] by P. Nevai and [30 by B. Simon for the role and various uses of Christoffel functions. Their asymptotics on the real line and on the unit circle have been well understood (34, 17, [18, [36, 6], 29, 32 31, 16], 44), but nothing was known about their asymptotic behavior on general curves (for a subarc of the circle and some special measures see [6]). In this paper we develop a method that can handle sets with boundary consisting of smooth Jordan curves. Our approach is based on approximation of general sets by lemniscates (level sets of polynomials), and it can simultaneously handle measures on curves and (area type measures) on domains. For example, it follows from our results that if $\mu$ is supported on a finite number of $C^{2}$ Jordan curves $\gamma$, then (provided the right hand side is positive and continuous)

$$
\lim _{n \rightarrow \infty} n \lambda_{n}(\mu, z)=\frac{d \mu(z)}{d \omega_{\gamma}}
$$


where $d \mu(z) / d \omega_{\gamma}$ denotes the Radon-Nikodym derivative of $\mu$ with respect to the equilibrium measure of $\gamma$.

Recall that Jordan curves are homeomorphic images of the unit circle. The bounded component of the complement of a Jordan curve is called a Jordan domain. If $\gamma$ is a union of rectifiable Jordan curves, then arc measure on $\gamma$ is denoted by $s_{\gamma}$.

Theorem 1.1. Let $\gamma$ be the union of finitely many rectifiable Jordan curves lying exterior to one another. Assume that $\mu$ is a Borel measure on $\gamma$ such that its Radon-Nikodym derivative $d \mu(t) / d s_{\gamma}$ with respect to $s_{\gamma}$ is positive $s_{\gamma}$-almost everywhere. Let $z_{0} \in \gamma, J$ be an open $C^{2}$ subarc of $\gamma$ containing $z_{0}$, and on $J$ let $\mu$ be absolutely continuous with respect to $s_{\gamma}$, and its Radon-Nikodym derivative $d \mu(t) / d s_{\gamma}$ continuous at $z_{0}$. Then

$$
\lim _{n \rightarrow \infty} n \lambda_{n}\left(\mu, z_{0}\right)=\frac{d \mu\left(z_{0}\right)}{d \omega_{\gamma}},
$$

where $\omega_{\gamma}$ is the equilibrium measure of $\gamma$. If the Radon-Nikodym derivative is continuous on $J$, then the convergence in (1.2) is uniform on compact subsets of $J$.

We remark that under the conditions of the theorem the Radon-Nikodym derivative on the right of (1.2) is also continuous at $z_{0}$. Furthermore, (1.2) can be written in the alternate form (see (3.5))

$$
\lim _{n \rightarrow \infty} n \lambda_{n}\left(\mu, z_{0}\right)=2 \pi \frac{d \mu\left(z_{0}\right)}{d s_{\gamma}}\left(\frac{\partial g_{\overline{\mathbf{C}} \backslash \gamma}\left(z_{0}, \infty\right)}{\partial \mathbf{n}}\right)^{-1},
$$

where $g_{\overline{\mathbf{C}} \backslash \gamma}\left(z_{0}, \infty\right)$ denotes the Green's function with pole at infinity associated with the unbounded component $\Omega$ of $\overline{\mathbf{C}} \backslash \gamma$ and where $\partial(\cdot) / \partial \mathbf{n}$ denotes the normal derivative in the direction of the inner normal of $\Omega$.

We also note that the condition $d \mu(t) / d s_{\gamma}>0 s_{\gamma}$-a.e. may be relaxed (see Theorem 1.2 below), but some type of condition of a similar nature is needed. For example, if $\mu$ vanishes on a subarc of $\gamma$, then (1.2) definitely fails.

We have stated Theorem 1.1 in a digestible form, but actually a more general result is true, of which Theorem 1.1 is a special case. Let $K=\operatorname{supp}(\mu)$ be the support of the measure $\mu$. We always assume that $K$ is compact and $\Omega$, the unbounded component of $\overline{\mathbf{C}} \backslash K$, is regular with respect to solving Dirichlet problems. The set $\overline{\mathbf{C}} \backslash \Omega$ is called the polynomial convex hull $\mathrm{Pc}(K)$ of $K$ (it is the union of $K$ with all the bounded components of $\overline{\mathbf{C}} \backslash K$ ), and the boundary $\partial \Omega$ of $\Omega$ is the so-called outer boundary of $K$. Points on the outer boundary is where we will consider the associated Christoffel functions (in $\Omega$ and inside $\operatorname{Pc}(K)$ its behavior is different; cf. Szegös' result at the beginning of the paper).

The measure $\mu$ is said to be in the Reg class (see [33, Theorem 3.2.3]) if the orthonormal polynomials with respect to $\mu$ have subexponential supremum norm on $K$, i.e. if

$$
\lim _{n \rightarrow \infty}\left\|p_{n}(\mu, \cdot)\right\|_{K}^{1 / n} \rightarrow 1 .
$$

This is equivalent to the fact that the $L^{2}(\mu)$ and $L^{\infty}(\mu)$ norms of polynomials are asymptotically the same in $n$-th root sense (see (6.29) ) or to the fact that $\lambda_{n}(\mu, z)^{1 / n} \rightarrow 1$ uniformly on the support of $\mu . \mu \in \mathbf{R e g}$ is a fairly weak condition 
on $\mu$; see 33 for general regularity criteria and different equivalent formulations of $\mu \in$ Reg. For example, if $d \mu(t) / d s_{\gamma}>0 s_{\gamma}$-almost everywhere as in Theorem 1.1, then $\mu \in \mathbf{R e g}$, so Theorem 1.1 is a special case of the following theorem, in which $\operatorname{cap}(K)$ stands for the logarithmic capacity of $K$ and $\omega_{K}$ for its equilibrium measure. The following theorem essentially says that Theorem 1.1 is true locally on arcs of the support; away from the arc considered the measure (and its support) can be fairly arbitrary.

Theorem 1.2. Assume that $\mu$ is in the $\mathbf{R e g}$ class such that its support $K$ satisfies $\operatorname{cap}(K)=\operatorname{cap}(\operatorname{Int}(\operatorname{Pc}(K)))$, where Int means two dimensional interior. Suppose that $z_{0} \in \partial \Omega$ and for some open disk $D$ with center at $z_{0}$ the intersection $D \cap K$ is a $C^{2}$ Jordan arc $J$, on which $\mu$ is absolutely continuous with respect to $s_{J}$ and its Radon-Nikodym derivative $d \mu(t) / d s_{J}$ is continuous at $z_{0}$. Then

$$
\lim _{n \rightarrow \infty} n \lambda_{n}\left(\mu, z_{0}\right)=\frac{d \mu\left(z_{0}\right)}{d \omega_{K}} .
$$

If the conditions are uniformly satisfied on $J$, then (1.5) uniformly holds on compact subsets of $J$.

Since $\operatorname{cap}(K)=\operatorname{cap}(\operatorname{Pc}(K))$, the condition of $K$ in the theorem says that $\operatorname{Pc}(K)$ is sufficiently thick in the sense that even its interior has the same capacity.

In what follows we shall often write $g_{\overline{\mathbf{C}} \backslash K}(z, \infty)$ for the Green's function $g_{\Omega}(z, \infty)$, where $\Omega$ is the unbounded component of $\overline{\mathbf{C}} \backslash K$. We shall prove Theorem 1.2 in the following equivalent form (see (3.5)):

$$
\lim _{n \rightarrow \infty} n \lambda_{n}\left(\mu, z_{0}\right)=2 \pi \frac{d \mu\left(z_{0}\right)}{d s_{J}}\left(\frac{\partial g_{\overline{\mathbf{C}} \backslash K}\left(z_{0}, \infty\right)}{\partial \mathbf{n}}\right)^{-1} .
$$

Theorem 1.2 gives asymptotics on arc-like measures under quite general conditions. The method that we shall use also gives asymptotics for Christoffel functions with respect to area-like measures. Orthogonal polynomials with respect to area measures go back to Carleman [1, who gave strong asymptotics for them in the case of a Jordan domain with analytic boundary curve. In this case one can read practically everything from the analytic extension of the external conformal map. For less smooth domains or for regions consisting of several components the situation is more difficult. There has been a recent burst of interest in this direction due partly to its relevance to planar region reconstruction ([9], 7, [15, 19]), but asymptotics of Christoffel functions on the boundary of the region have not been known or investigated except for the paper [10, where lower and upper bounds were given for them.

Let $A$ denote area measure. A new feature in this case is that on the boundary the order of the Christoffel function will be $1 / n^{2}$ (as opposed to the order $1 / n$ above), which is quite natural, since typically a minimizing polynomial for $\lambda_{n}(\mu, z)$ is "small outside a $1 / n$-neighborhood of $z$ ", and with respect to area measure the square integral of it will be of the order $1 / n^{2}$. For example, for a set $K$ enclosed by finitely many $C^{2}$ Jordan curves the Christoffel functions with respect to area 
measure $d A$ have asymptotics

$$
\lim _{n \rightarrow \infty} n^{2} \lambda_{n}(d A, z)=2 \pi\left(\frac{\partial g_{\Omega}(z, \infty)}{\partial \mathbf{n}}\right)^{-2}
$$

uniformly on the boundary of $K$. A more precise result is

Theorem 1.3. Suppose that $K$ is a compact set and $\mu$ is a measure on $K$ of the form $d \mu=W d A$ with some continuous $W$ such that

$$
\operatorname{cap}(\{z \mid W(z)>0\} \cap \operatorname{Int}(K))=\operatorname{cap}(K) .
$$

Suppose that $z_{0} \in \partial \Omega$, and for some open disk $D$ with center at $z_{0}$ the intersection $D \cap \partial K$ is a $C^{2}$ Jordan arc J. Then

$$
\lim _{n \rightarrow \infty} n^{2} \lambda_{n}\left(\mu, z_{0}\right)=2 \pi W\left(z_{0}\right)\left(\frac{\partial g_{\overline{\mathbf{C} \backslash K}}\left(z_{0}, \infty\right)}{\partial \mathbf{n}}\right)^{-2} .
$$

If the conditions are uniformly satisfied on $J$, then (1.8) uniformly holds on compact subsets of $J$.

This is again a special case of

Theorem 1.4. Let $\mu$ be a Borel measure in the $\mathbf{R e g}$ class such that $K=\operatorname{supp}(\mu)$ is a compact set with $\operatorname{cap}(K)=\operatorname{cap}(\operatorname{Int}(\operatorname{Pc}(K)))$. Suppose that $z_{0} \in \partial \Omega$, and for some open disk $D$ with center at $z_{0}$ the intersection $D \cap \partial K$ is a $C^{2}$ Jordan arc J. Assume that in $K \cap D \mu$ is of the form $d \mu=W d A$ with some $W$ that is continuous at $z_{0}$. Then (1.8) holds. If $W$ is continuous on $J$, then (1.8) holds uniformly on compact subsets of $J$.

We mention that (1.7) was also announced in 10 by B. Gustafsson, M. Putinar, E. B. Saff and N. Stylianopoulos in the case where $K$ is a Jordan domain with analytic boundary. For more than one component lower and upper bounds for $n^{2} \lambda_{n}(d A, z)$ were also given there (see also the manuscript [11]). The emphasis in 10. was on the general theory of orthogonal polynomials with respect to area measure (Bergman polynomials) on the union of finitely many Jordan domains, and the method of that paper is totally different from what we use below.

The idea of our proofs is to verify the results for lemniscate sets (level sets of polynomials) using polynomial mappings. We shall see that we can well approximate our sets (supports) by lemniscates, and that will allow us to prove the theorems from their lemniscate special case. This will be achieved by a delicate extension of Hilbert's lemniscate theorem proved in [20] which roughly claims that one can insert a lemniscate in between touching Jordan curves in such a way that the normal derivative of the corresponding Green's functions is also close (see Theorem 2.3). Thus, in a sense, the basis of the proof is the application of polynomial mappings, so it resembles the reasonings in [37] and [36.

The proof for area-like measures is technically much simpler than for measures on curves. Therefore we shall prove the former ones first. The reason for the area case being simple is that inner and outer exhaustions using lemniscate domains are sufficient to derive lower and upper bounds, respectively. However, in the curve case there is no lemniscate which precisely matches the curve, and this adds another level of difficulty. Also, although Theorem 1.3 is a special case of Theorem 1.4, that is not easy to see; hence we shall prove both of them (skipping, of course, the common parts in the second proof). 
The paper is organized as follows. First we prove some facts on lemniscates and lemniscate domains. Then we verify some elementary results on Green's functions and equilibrium measures. In Section 4 we present the construction of some fast decreasing polynomials that will be used throughout the proofs. The proof of Theorems 1.3 1.4 will be presented in Section 5, and that of Theorems 1.1 1.2 in Section 6. Finally, we establish some simple Markov and Bernstein type inequalities that also play an essential role.

In conclusion, we mention that the case when $\mu$ is supported on Jordan arcs (even a single smooth arc) is still open.

\section{A FEW ELEMENTARY FACTS ABOUT LEMNISCATES AND THEIR DOMAINS}

Let $\mathcal{C}=\{w|| w \mid=1\}$ and $\Delta=\{w|| w \mid<1\}$ be the unit circle and unit disk, respectively.

A lemniscate $\sigma$ is the level line of a polynomial, i.e.

$$
\sigma=T_{N}^{-1}(\mathcal{C})=\left\{z|| T_{N}(z) \mid=1\right\}
$$

where $T_{N}$ is a polynomial of exact degree $N$. Although $\sigma$ may have several $(\leq N)$ components, we shall call the set $L=L_{\sigma}=T_{N}^{-1}(\bar{\Delta})=\operatorname{Pc}(\sigma)$ that $\sigma$ encloses a closed lemniscate domain. In what follows we shall always assume that $\sigma$ consists of disjoint Jordan curves (homeomorphic images of the unit circle). Then $T_{N}^{\prime}(z) \neq 0$ on $\sigma$, and $\sigma$ consists of finitely many analytic Jordan curves.

The Green's function of $\overline{\mathbf{C}} \backslash L$ with pole at infinity is

$$
g_{\overline{\mathbf{C}} \backslash L}(z, \infty)=\frac{1}{N} \log \left|T_{N}(z)\right|
$$

(just check the defining properties of the Green's function for the right hand side; see [26. Definition 4.4.1]), and its normal derivative at $z \in \partial L$ with respect to the inner normal of $\overline{\mathbf{C}} \backslash L$ (outer normal to $L$ ) is

$$
\frac{\partial g_{\overline{\mathbf{C} \backslash L}}(z, \infty)}{\partial \mathbf{n}}=\frac{1}{N}\left|T_{N}^{\prime}(z)\right| .
$$

Indeed, in verifying this we may assume that $z=0, T_{N}(0)=1$ and $\mathbf{n}$ is pointing in the direction of the positive $x$ axis. Then the boundary of $L$ has a vertical tangent line at 0 , which is mapped by $T_{N}$ into the boundary of $\Delta$ with vertical tangent line at $T_{N}(0)=1$. Hence the conformality of $T_{N}$ gives $T_{N}^{\prime}(0)>0$, and

$$
\begin{aligned}
\frac{\partial g_{\overline{\mathbf{C} \backslash L}}(0, \infty)}{\partial \mathbf{n}} & =\left.\frac{d g_{\overline{\mathbf{C} \backslash L}}(x, \infty)}{d x}\right|_{x=0}=\left.\frac{1}{N} \frac{d \log \left|T_{N}(x)\right|}{d x}\right|_{x=0} \\
& =\left.\Re \frac{1}{N} \frac{d \log T_{N}(x)}{d x}\right|_{x=0}=\Re \frac{1}{N} \frac{T_{N}^{\prime}(0)}{T_{N}(0)}=\frac{1}{N} T_{N}^{\prime}(0),
\end{aligned}
$$

since $T_{N}(0)=1$.

In what follows we write $d A=d A(z)=d x d y(z=x+i y)$, so that

$$
\int \cdots d A=\iint \cdots d x d y
$$

Define for some $\rho<1$ close to 1 the sets

$$
\Delta_{\rho}=\{w|| w \mid<\rho\}, \quad \Delta_{\rho}^{*}=\{w|\rho<| w \mid<1\}, \quad \tilde{L}_{\rho}=T^{-1}\left(\Delta_{\rho}\right)
$$


and

$$
L_{\rho}^{*}=T^{-1}\left(\Delta_{\rho}^{*}\right),
$$

of which $L_{\rho}^{*}$ will be our first concern. For $\rho<1$ lying close to 1 this $L_{\rho}^{*}$ lies close to the boundary $\partial L$, and we assume $\rho<1$ so large that in $L_{\rho}^{*}$ we have $T_{N}^{\prime}(z) \neq 0$, i.e. in $L_{\rho}^{*}$ the mapping $w=T_{N}(z)$ is locally $1-$ to -1 . Of course, on $L_{\rho}^{*}$ it is globally $N$-to -1 . The Jacobian of this mapping is $\left|T_{N}^{\prime}(z)\right|^{2}$, i.e. $d A(w)=\left|T_{N}^{\prime}(z)\right|^{2} d A(z)$, and hence (say for continuous $g$ )

$$
\int_{L} g\left(T_{N}(z)\right)\left|T_{N}^{\prime}(z)\right|^{2} d A(z)=N \int_{\bar{\Delta}} g(w) d A(w) .
$$

The set $T_{N}^{-1}([-1,-1+\rho])$ cuts $L_{\rho}^{*}$ into $N$ connected components $G_{1}, \ldots, G_{N}$, each of which is mapped by $T_{N}$ into $\Delta_{\rho}^{*} \backslash[-1,-1+\rho]$ in a 1-to-1 fashion. As we have just mentioned, the Jacobian of the mapping $w=T_{N}(z)$ is $\left|T_{N}^{\prime}(z)\right|^{2}$, hence

$$
\int_{G_{j}} g\left(T_{N}(z)\right)\left|T_{N}^{\prime}(z)\right|^{2} d A(z)=\int_{\Delta_{\rho}^{*}} g(w) d A(w) .
$$

Let $T_{N, j}^{-1}$ be the inverse of the restriction of $T_{N}$ to $G_{j}$, i.e. $T_{N, j}^{-1}$ is the branch of $T^{-1}$ that maps $\Delta_{\rho}^{*} \backslash[-1,-1+\rho]$ onto $G_{j}$. The Jacobian of $h_{i, j}(z)=T_{N, j}^{-1}\left(T_{N}(z)\right)$ mapping $G_{i}$ onto $G_{j}$ is

$$
\left|\frac{1}{T_{N}^{\prime}\left(T_{N, j}^{-1}\left(T_{N}(z)\right)\right)} \cdot T_{N}^{\prime}(z)\right|^{2}=\left|\frac{T_{N}^{\prime}(z)}{T_{N}^{\prime}\left(h_{i j}(z)\right)}\right|^{2} .
$$

For a $z \in L_{\rho}^{*}$ (say for $T_{N}(z) \notin[-1,-1+\rho]$ ) let $z_{j}=T_{N, j}^{-1}\left(T_{N}(z)\right)$. Then this $z_{j}$ belongs to $G_{j}$, and each $z_{j}$ is a function of any other $z_{i}: z_{j}=h_{i j}\left(z_{i}\right)$. Hence, from what we have just said about the Jacobian, it follows that

$$
d A\left(z_{j}\right)=\left|\frac{T_{N}^{\prime}\left(z_{i}\right)}{T_{N}^{\prime}\left(h_{i j}\left(z_{i}\right)\right)}\right|^{2} d A\left(z_{i}\right)=\left|\frac{T_{N}^{\prime}\left(z_{i}\right)}{T_{N}^{\prime}\left(z_{j}\right)}\right|^{2} d A\left(z_{i}\right),
$$

and so (say for a continuous $f$ )

$$
\begin{aligned}
\int_{G_{j}} f\left(z_{i}\right)\left|T_{N}^{\prime}\left(z_{j}\right)\right|^{2} d A\left(z_{j}\right) & =\int_{G_{i}} f\left(z_{i}\right)\left|T_{N}^{\prime}\left(h_{i j}\left(z_{i}\right)\right)\right|^{2}\left|\frac{T_{N}^{\prime}\left(z_{i}\right)}{T_{N}^{\prime}\left(h_{i j}\left(z_{i}\right)\right)}\right|^{2} d A\left(z_{i}\right) \\
& =\int_{G_{i}} f\left(z_{i}\right)\left|T_{N}^{\prime}\left(z_{i}\right)\right|^{2} d A\left(z_{i}\right) .
\end{aligned}
$$

Therefore,

$$
\int_{G_{j}}\left(\sum_{i=1}^{N} f\left(z_{i}\right)\right)\left|T_{N}^{\prime}\left(z_{j}\right)\right|^{2} d A\left(z_{j}\right)=\int_{L_{\rho}^{*}} f(z)\left|T_{N}^{\prime}(z)\right|^{2} d A(z),
$$

and finally

$$
\int_{L_{\rho}^{*}}\left(\sum_{i=1}^{N} f\left(z_{i}\right)\right)\left|T_{N}^{\prime}(z)\right|^{2} d A(z)=N \int_{L_{\rho}^{*}} f(z)\left|T_{N}^{\prime}(z)\right|^{2} d A(z) .
$$

Next let $s_{\sigma}$ denote the arc length measure on the lemniscate $\sigma$. In what follows we consider $T_{N}$ only on $\sigma$. The set $T_{N}^{-1}(-1)$ cuts $\sigma$ into $N \operatorname{arcs} \sigma_{1}, \ldots, \sigma_{N}$. Since 
along the curve $\sigma$ the Jacobian of the mapping $w=T_{N}(t)(t \in \sigma)$ is $\left|T_{N}^{\prime}(t)\right|$, the formula (2.6) now takes the form

$$
d s_{\sigma}\left(t_{j}\right)=\left|\frac{T_{N}^{\prime}\left(t_{i}\right)}{T_{N}^{\prime}\left(h_{i j}\left(t_{i}\right)\right)}\right| d s_{\sigma}\left(t_{i}\right)=\left|\frac{T_{N}^{\prime}\left(t_{i}\right)}{T_{N}^{\prime}\left(t_{j}\right)}\right| d s_{\sigma}\left(t_{i}\right),
$$

and the analogue of (2.7) is

$$
\int_{\sigma_{j}} f\left(t_{i}\right)\left|T_{N}^{\prime}\left(t_{j}\right)\right| d s_{\sigma}\left(t_{j}\right)=\int_{\sigma_{i}} f\left(t_{i}\right)\left|T_{N}^{\prime}\left(t_{i}\right)\right| d s_{\sigma}\left(t_{i}\right)
$$

Hence

$$
\int_{\sigma_{j}}\left(\sum_{i=1}^{N} f\left(t_{i}\right)\right)\left|T_{N}^{\prime}\left(t_{j}\right)\right| d s_{\sigma}\left(t_{j}\right)=\int_{\sigma} f(t)\left|T_{N}^{\prime}(t)\right| d s_{\sigma}(t)
$$

and

$$
\int_{\sigma}\left(\sum_{i=1}^{N} f\left(t_{i}\right)\right)\left|T_{N}^{\prime}(t)\right| d s_{\sigma}(t)=N \int_{\sigma} f(t)\left|T_{N}^{\prime}(t)\right| d s_{\sigma}(t) .
$$

Since $T_{N}$ maps $\sigma$ into the unit circle $\mathcal{C}$ in an $N$-to- 1 fashion and the Jacobian of the mapping $\theta=T_{N}(t)$ is $\left|T_{N}^{\prime}(t)\right|$, it follows that

$$
\int_{\sigma_{j}} g\left(T_{N}(t)\right)\left|T_{N}^{\prime}(t)\right| d s_{\sigma}(t)=\int_{\mathcal{C}} g(\theta) d s_{\mathcal{C}}(\theta)=\int_{0}^{2 \pi} g\left(e^{i \tau}\right) d \tau
$$

and therefore

$$
\int_{\sigma} g\left(T_{N}(t)\right)\left|T_{N}^{\prime}(t)\right| d s_{\sigma}(t)=N \int_{\mathcal{C}} g(\theta) d s_{\mathcal{C}}(\theta)=N \int_{0}^{2 \pi} g\left(e^{i \tau}\right) d \tau .
$$

Next we show that lemniscate domains can well approximate quite general sets. Hilbert's lemniscate theorem [26, Theorem 5.5.8] says the following. Let $\gamma_{j}, \Gamma_{j}$, $j=1, \ldots, m$, be Jordan curves, $\gamma_{j}$ lying interior to $\Gamma_{j}$ and the $\Gamma_{j}$ 's lying exterior to one another, and set $\gamma^{*}=\bigcup_{j} \gamma_{j}, \Gamma^{*}=\bigcup_{j} \Gamma_{j}$. Then there is a lemniscate $\sigma$ contained in the interior of $\Gamma^{*}$ that contains $\gamma^{*}$ in its interior, i.e. $\sigma$ separates $\gamma^{*}$ and $\Gamma^{*}$ in the sense that it separates each $\gamma_{j}$ from the corresponding $\Gamma_{j}$. This is not enough for us; we need the following sharpening from [20, Theorem 1.1].

Theorem 2.1. Let $\gamma^{*}=\bigcup_{j=1}^{m} \gamma_{j}$ and $\Gamma^{*}=\bigcup_{j=1}^{m} \Gamma_{j}$ be as above, and let $\gamma^{*}$ and $\Gamma^{*}$ touch each other at a point $P$ in a neighborhood of which both curves are twice continuously differentiable and the curvatures of $\gamma^{*}$ and $\Gamma^{*}$ are different at $P$. Then there is a lemniscate $\sigma$ that separates $\gamma^{*}$ and $\Gamma^{*}$ and touches both $\gamma^{*}$ and $\Gamma^{*}$ at $P$. Furthermore, $\sigma$ lies strictly in between $\gamma^{*}$ and $\Gamma^{*}$ except for the point $P$ and has precisely one connected component in between each $\gamma_{j}$ and $\Gamma_{j}, j=1, \ldots, m$; these $m$ components are Jordan curves. The curvature of $\sigma$ at $P$ is different from the curvatures of $\gamma$ and $\Gamma$ at $P$.

Actually, this result is true for finitely many touching points, in which case there is still a separating lemniscate (see [20, Theorem 1.1]).

We are going to use this in the following form (see [20, Theorem 1.2]).

Theorem 2.2. Let $\gamma$ consist of finitely many Jordan curves lying exterior to one another, let $K=K_{\gamma}=\operatorname{Pc}(\gamma)$ be the "closed domain" enclosed by $\gamma$, let $P \in \gamma$, and assume that in a neighborhood of $P$ the curve $\gamma$ is $C^{2}$ smooth. Then for every $\varepsilon>0$ there is a lemniscate $\sigma$ consisting of Jordan curves such that $\sigma$ touches $\gamma$ at $P, \sigma$ 
contains $K$ in its interior except for the point $P$, every component of $\sigma$ contains precisely one component of $\gamma$ and (with $L=L_{\sigma}$ )

$$
\frac{\partial g_{\overline{\mathbf{C} \backslash K}}(P, \infty)}{\partial \mathbf{n}} \leq \frac{\partial g_{\overline{\mathbf{C} \backslash L}}(P, \infty)}{\partial \mathbf{n}}+\varepsilon,
$$

where $\partial(\cdot) / \partial \mathbf{n}$ denotes (outward) normal derivative.

In a similar manner, for every $\varepsilon>0$ there is a lemniscate $\sigma$ consisting of Jordan curves such that $\sigma$ touches $\gamma$ at $P, \sigma$ lies strictly inside $K$ except for the point $P$, $\sigma$ has precisely one component lying inside every component of $\gamma$, and

$$
\frac{\partial g_{\overline{\mathbf{C} \backslash L}}(P, \infty)}{\partial \mathbf{n}} \leq \frac{\partial g_{\overline{\mathbf{C} \backslash K}}(P, \infty)}{\partial \mathbf{n}}+\varepsilon .
$$

Furthermore, this is also true if $K$ is not the whole "closed domain" $K_{\gamma}=\operatorname{Pc}(\gamma)$ enclosed by $\gamma$, but for some $\delta>0$ we have that

$$
K_{\gamma} \cap\{z \mid \operatorname{dist}(z, \gamma)<\delta\} \subseteq K .
$$

Note that in (2.15) we have

$$
\frac{\partial g_{\overline{\mathbf{C}} \backslash L}(P, \infty)}{\partial \mathbf{n}} \leq \frac{\partial g_{\overline{\mathbf{C}} \backslash K}(P, \infty)}{\partial \mathbf{n}}
$$

because $K \subset L$, while in (2.16) we have

$$
\frac{\partial g_{\overline{\mathbf{C} \backslash K}}(P, \infty)}{\partial \mathbf{n}} \leq \frac{\partial g_{\overline{\mathbf{C} \backslash L}}(P, \infty)}{\partial \mathbf{n}}
$$

because $L \subset K$.

Indeed, this was a consequence of Theorem 2.3 by appropriately choosing $\Gamma^{*}$ for $\gamma^{*}=\gamma$ (respectively $\gamma^{*}$ for $\left.\Gamma^{*}=\gamma\right)$. We shall also need the following sharper form.

Theorem 2.3. Let $\gamma$ consist of finitely many Jordan curves lying exterior to one another, let $Z \in \gamma$ and assume that for some disk $D$ with center at $Z$ the intersection $D \cap \gamma$ is a $C^{2}$ arc. Then for $P \in \gamma$ lying sufficiently close to $Z$ the $\sigma=\sigma_{P}$ in (2.15) (or (2.16) ) can be a translated-rotated copy of a fixed lemniscate, say of $\sigma_{Z}$.

Proof. Denote the open disk of radius $r$ about the point $P$ by $\Delta_{r}(P)$. Choose a $\Gamma^{*}$ as in Theorem 2.1 that touches $\gamma^{*}=\gamma$ at $Z$, has $C^{2}$ boundary near $Z$ and its curvature at $P$ is different from the curvature of $\gamma$. Then there is a separating lemniscate $\sigma=\sigma_{Z}$ as in Theorem 2.1 for which (2.15) is also true at $P=Z$ with $\varepsilon / 2$ instead of $\varepsilon$. Choose a small radius $r$ such that in $\Delta_{r}(Z)$ the curvature of $\gamma$ (more precisely, the signed curvature seen from the outside) is larger than the curvature of $\Gamma$ (it must be larger, since $\gamma$ lies inside $\Gamma$ ), and the curvature of $\sigma$ lies in between these curvatures. Thus, there are $a<A$ such that the curvature of $\gamma$ is larger than $A$ and the curvature of $\Gamma$ is smaller than $a$, while the curvature of $\sigma$ lies in the interval $(a, A)$ at every point of $\Delta_{r}(Z) \cap \gamma$. Let $\sigma_{P}$ be the translated/rotated copy of $\sigma$ where the point $Z$ is moved to $P$ and $\sigma_{P}$ touches $\gamma$ at $P$ (actually, there are two such copies, but it is obvious which one we take). Since $K$ lies strictly inside $\sigma$ except for the point $Z$, if $\delta<r / 6$ is small and $|P-Z|<\delta$, then $K \backslash \Delta_{r / 3}(P)$ lies strictly inside $\sigma_{P}$. At $P$ the two curves $\gamma$ and $\sigma_{P}$ touch each other, and the curvature of $\sigma_{P}(<A)$ is strictly smaller than the curvature of $\gamma$ $(>A)$ in $\Delta_{r / 3}(P)$. Hence, by Blaschke's rolling theorem (3, Ch. 4., Section 24, subsection II] or see [20, Lemma 6.3] for a local version) $\gamma \cap \Delta_{r / 3}(P)$ lies inside $\sigma_{P}$; more precisely $K \cap \Delta_{r / 3}(P) \subset L_{\sigma_{P}} \cap \Delta_{r / 3}(P)$ (here, as before, $L_{\sigma_{P}}=\operatorname{Pc}\left(\sigma_{P}\right)$ 
is the closed lemniscate domain enclosed by $\sigma_{P}$ ). These together show that $K$ lies interior to $\sigma_{P}$ (except for the point $P$ ).

Finally, since the normal derivative of the Green's function $g_{\overline{\mathbf{C}} \backslash K}(z, \infty)$ is continuous in a neighborhood of $Z$ (see the last statement in Theorem 3.2), we may choose $r$ so small that in $\Delta_{r}(Z)$ the normal derivative does not change more than $\varepsilon / 2$. Then (2.15) also holds true for $\sigma=\sigma_{P}$ when $P \in \Delta_{r}(Z)$, since it was true for $\sigma=\sigma_{Z}$ with $\varepsilon / 2$ instead of $\varepsilon$, and the normal derivatives at $P$ and at $Z$ differ by at most $\varepsilon / 2$.

The proof for inner lemniscate and for (2.16) is the same; just set $\Gamma^{*}=\gamma$ and select an appropriate $\gamma^{*}$ inside.

\section{EqUilibrium MEASURES AND GREEN'S FUnCTIONS}

In this section we prove some simple results concerning equilibrium measures and Green's functions that are more or less folklore, but we need them in the localized form stated below.

Let $K$ be a compact set on the plane with positive logarithmic capacity cap $(K)$, $g_{\overline{\mathbf{C}} \backslash K}(z, \infty)$ the Green's function with pole at infinity of the unbounded component $\Omega$ of $\overline{\mathbf{C}} \backslash K$, and $\omega_{K}$ the equilibrium measure of $K$. For all these concepts see [26]. We shall also use the logarithmic potential

$$
U^{\omega}(z)=\int \log \frac{1}{|z-t|} d \omega(t)
$$

of a measure $\omega$ with compact support on $\overline{\mathbf{C}}$. With this we have the representation (see e.g. [26, p. 107])

$$
g_{\overline{\mathbf{C} \backslash K}}(z, \infty)=-U^{\omega_{K}}(z)-\log \operatorname{cap}(K) .
$$

Theorem 3.1. If $\gamma$ is a Jordan curve, $K$ is the closed domain enclosed by $\gamma$ and $\Phi$ is the conformal map from $\overline{\mathbf{C}} \backslash K$ onto the exterior of the unit disk, then $\Phi$ extends to a homeomorphism of $\gamma$ onto the unit circle $\mathcal{C}$ and $\omega_{K}$ is the pull-back of the normalized arc measure on $\mathcal{C}$ under $\Phi$; i.e. for any Borel set $E$ we have $\omega_{K}(E)=|\Phi(E)| / 2 \pi$, where $|\cdot|$ indicates arc length on $\mathcal{C}$.

Proof. That $\Phi$ extends to a homeomorphism of the boundaries is the classical result of Caratheodory; see [40, II, Theorem VII, (10.9)] or [25, Theorem 2.6].

Let $\omega(E)=|\Phi(E)| / 2 \pi$ be the pull-back measure in question, and consider its logarithmic potential at $z=\Phi^{-1}(w) \in \overline{\mathbf{C}} \backslash K$ :

$$
\begin{aligned}
U^{\omega}(z) & =\int_{\gamma} \log \frac{1}{|z-t|} d \omega(t)=\frac{1}{2 \pi} \int_{0}^{2 \pi} \log \frac{1}{\left|\Phi^{-1}(w)-\Phi^{-1}\left(e^{i \tau}\right)\right|} d \tau \\
& =\frac{1}{2 \pi} \int_{0}^{2 \pi} \log \frac{\left|w-e^{i \tau}\right|}{\left|\Phi^{-1}(w)-\Phi^{-1}\left(e^{i \tau}\right)\right|} d \tau+\frac{1}{2 \pi} \int_{0}^{2 \pi} \log \frac{1}{\left|w-e^{i \tau}\right|} d \tau
\end{aligned}
$$

The last term is $-\log |w|=-\log |\Phi(z)|$, which is equal to the logarithmic potential of $\omega_{K}$ modulo a constant (see the formula (3.2) and use the fact that $\log |\Phi(z)|$ is the Green's function of $\mathbf{C} \backslash K$ with pole at infinity). The function

$$
\log \frac{|w-z|}{\left|\Phi^{-1}(w)-\Phi^{-1}(z)\right|}
$$


is harmonic in $z$ on the exterior of the unit disk (including the point infinity) and is continuous on the boundary. Hence its average over the unit circle is equal to its value at infinity, i.e. the first term on the right in (3.3) is $\left.\log \left|z / \Phi^{-1}(z)\right|\right|_{z=\infty}$. All in all, it follows from these facts and from (3.2) that $U^{\omega}(z)-U^{\omega_{K}}(z)$ is constant on $\overline{\mathbf{C}} \backslash K$. Then this constant has to be 0 , since $U^{\omega}(z)-U^{\omega_{K}}(z)$ tends to 0 az $|z| \rightarrow \infty$ (note that both $\omega$ and $\omega_{K}$ are probability measures on $\gamma$ ). Hence, $U^{\omega}(z) \equiv U^{\omega_{K}}(z)$ in $\overline{\mathbf{C}} \backslash K$, and $\omega=\omega_{K}$ follows from Carleson's unicity theorem [28, Theorem II.4.3].

Theorem 3.2. Let $K$ be a compact set on the plane, $\Omega$ the unbounded component of $\overline{\mathbf{C}} \backslash K$, and $J$ a $C^{2}$ arc on the boundary of $\Omega$ such that $J=\partial \Omega \cap O$ with some open set $O$. Then on $J$

$$
d \omega_{K}(t)=\frac{1}{2 \pi} \frac{\partial g_{\overline{\mathbf{C}} \backslash K}(t, \infty)}{\partial \mathbf{n}} d s_{J}(t),
$$

where $\partial(\cdot) / \partial \mathbf{n}$ denotes the normal derivative with respect to the inner normal to $\Omega$ on $J$, and $d s_{J}(t)$ denotes integration with respect to the arc measure on $J$.

Furthermore, $\partial g_{\overline{\mathbf{C} \backslash K}}(t, \infty) / \partial \mathbf{n}$ is continuous on $J$.

In particular, we get the identity

$$
\frac{d \omega_{K}(t)}{d s}=\frac{1}{2 \pi} \frac{\partial g_{\overline{\mathbf{C} \backslash K}}(t, \infty)}{\partial \mathbf{n}}
$$

for the Radon-Nikodym derivative of $\omega_{K}$ with respect to arc length.

Proof. For $\Omega$ with a $C^{2}$ boundary, this is [28, Theorem 4.10] if we note that the balayage $\widehat{\delta_{\infty}}$ of the Dirac mass $\delta_{\infty}$ at infinity out of $\Omega$ is the equilibrium measure $\omega_{K}([26$, Theorem 4.3.14]).

To obtain the general case, let us exhaust $\Omega$ by an increasing sequence $\left\{\omega_{n}\right\}$ of (connected) domains with $C^{2}$ boundary such that some given closed subarc $J_{1}$ of $J$ lies on each $\partial \omega_{n}$, and for some neighborhood $O_{1}$ of $J_{1}$ we also have $O_{1} \cap \Omega \subset \omega_{n}$ for all $n$. Then $g_{\Omega_{n}}(z, \infty) \rightarrow g_{\Omega}(z, \infty)$ as $n \rightarrow \infty$ for all $z \in \Omega$ (see [26, Theorem 4.4.6]). Now we can invoke Lemma 3.3 below to conclude that

$$
\frac{\partial g_{\Omega_{n}}(t, \infty)}{\partial \mathbf{n}} \rightarrow \frac{\partial g_{\Omega}(t, \infty)}{\partial \mathbf{n}}
$$

uniformly in $t \in J_{2}$, where $J_{2} \subset J_{1}$ is a any closed subarc of $J_{1}$ not having common endpoints with $J_{1}$.

On the other hand, if $K_{n}=\overline{\mathbf{C}} \backslash \Omega_{n}$, then by Helly's theorem we can select a weak* convergent subsequence $\left\{\omega_{K_{n}}\right\}_{n \in \mathcal{N}}$ from $\omega_{K_{n}}$; say it converges to some measure $\rho$. It is clear that $\rho$ is a unit Borel measure on $\partial \Omega$ such that its logarithmic potential at a point $z \in \Omega$ is the limit of the logarithmic potentials

$$
U^{\mu_{K_{n}}}(z)=-g_{\Omega_{n}}(z, \infty)-\log \operatorname{cap}\left(K_{n}\right), \quad n \in \mathcal{N} .
$$

We have just seen that the limit of $g_{\Omega_{n}}(z, \infty)$ is $g_{\Omega}(z, \infty)$, and, since $K_{n}$ is a nested sequence, we also have by [26, Theorem 5.1.3,a] the relation $\operatorname{cap}\left(K_{n}\right) \rightarrow$ $\operatorname{cap}(\overline{\mathbf{C}} \backslash \Omega)=\operatorname{cap}(K)$. Thus,

$$
U^{\rho}(z)=-g_{\Omega}(z, \infty)-\log \operatorname{cap}(K)=U^{\omega_{K}}(z), \quad z \in \Omega,
$$

and we can deduce $\rho=\omega_{K}$ from Carleson's unicity theorem [28, Theorem II.4.3]. Since this can be said for any convergent subsequence selected from any subsequence of $\left\{\omega_{K_{n}}\right\}$, we can conclude that $\omega_{K_{n}} \rightarrow \omega_{K}$ in the weak* sense. 
Now since on $J_{1}$ we have

$$
d \omega_{K_{n}}(t)=\frac{1}{2 \pi} \frac{\partial g_{\overline{\mathbf{C} \backslash K_{n}}}(t, \infty)}{\partial \mathbf{n}} d s_{\partial \omega_{n}}(t),
$$

where the left hand side converges in the weak* topology to the left hand side in (3.4) and the right hand side converges uniformly on $J_{1}$ to the right hand side of (3.4), we get the equality (3.4) inside $J_{1}$. In fact, let $\varphi$ be an arbitrary continuous test function with compact support inside $O_{1}$ (see the beginning of the proof) such that $\operatorname{supp}(\varphi) \cap \partial \Omega \subset J_{1}$. Then

$$
\int \varphi d \omega_{K}=\lim _{n \rightarrow \infty} \int \varphi d \omega_{K_{n}}
$$

because of weak* convergence, and

$$
\int \varphi(t) \frac{\partial g_{\overline{\mathbf{C}} \backslash K}(t, \infty)}{\partial \mathbf{n}} d s_{J}(t)=\lim _{n \rightarrow \infty} \int \varphi(t) \frac{\partial g_{\overline{\mathbf{C}} \backslash K_{n}}(t, \infty)}{\partial \mathbf{n}} d s_{J}(t)
$$

because of uniform convergence on $J_{1}$. Thus, (3.6) and

$$
\left.s_{J}\right|_{O_{1}}=\left.s_{\partial \Omega_{n}}\right|_{O_{1}}
$$

give

$$
\int \varphi d \omega_{K}=\int \varphi(t) \frac{\partial g_{\overline{\mathbf{C}} \backslash K}(t, \infty)}{\partial \mathbf{n}} d s(t),
$$

and since this is true for all $\varphi$ as above, we can conclude (3.4) inside $J_{1}$.

To prove the last statement, let $J_{1}$ be a closed subarc of $J$ and select a bounded $C^{2}$-domain $G$ inside $\Omega$ such that it contains on its boundary $J_{1}$. A $C^{2}$ conformal map $\Phi$ takes $G$ onto the unit disk, and $g_{\overline{\mathbf{C}} \backslash K}\left(\Phi^{-1}(w), \infty\right)$ is harmonic on the unit disk with identically 0 boundary value inside $\Phi\left(J_{1}\right)$. A simple inspection based on Poisson's formula then shows the continuity of the normal derivative (first on the unit disk and then carry this back to $G$ ).

We shall frequently use the following

Lemma 3.3. Let $\Omega$ be a domain with compact boundary, $\infty \in \Omega, D$ an open disk with center on $\partial \Omega$ and assume that $\partial \Omega \cap \bar{D}$ is a $C^{2}$ arc. Suppose also that $\left\{\Omega_{n}\right\}$ are domains such that $D \cap \Omega=D \cap \Omega_{n}$ for all $n$ and one of the following conditions holds:

(i) $\Omega_{n} \subset \Omega_{n+1}$ for all $n$, and $\bigcup \Omega_{n}=\Omega$,

(ii) $\Omega_{n+1} \subset \Omega_{n}$ for all $n$, and $\bigcap \Omega_{n}=\Omega$,

(iii) $\Omega \subset \Omega_{n}$ for all $n$, and $\operatorname{cap}\left(\overline{\mathbf{C}} \backslash \Omega_{n}\right) \rightarrow \operatorname{cap}(\overline{\mathbf{C}} \backslash \Omega)$ as $n \rightarrow \infty$.

Then

$$
\frac{\partial g_{\Omega_{n}}(z, \infty)}{\partial \mathbf{n}} \rightarrow \frac{\partial g_{\Omega}(z, \infty)}{\partial \mathbf{n}}
$$

uniformly on compact subsets of $D \cap \partial \Omega$, where $\partial(\cdot) / \partial \mathbf{n}$ denotes normal derivative with respect to the inner normal to $\Omega$.

Proof. Note that by [26, Theorem 5.1.3] we have in either case cap $\left(\overline{\mathbf{C}} \backslash \Omega_{n}\right) \rightarrow$ $\operatorname{cap}(\overline{\mathbf{C}} \backslash \Omega)$. 
Assume, for example, (ii). Let $\overline{D_{1}} \subset D$ be a closed disk with the same center as $D$, and choose a bounded simply connected domain $G$ in $\Omega$ with $C^{2}$ boundary such that $G$ contains $\Omega \cap \overline{D_{1}}$ and $\partial G \backslash \overline{D_{1}} \subseteq \Omega$. Since

$$
g_{\Omega_{n}}(z, \infty)-g_{\Omega}(z, \infty) \geq 0
$$

and

$$
g_{\Omega_{n}}(z, \infty)-\left.g_{\Omega}(z, \infty)\right|_{z=\infty}=\log \operatorname{cap}(\overline{\mathbf{C}} \backslash \Omega)-\log \operatorname{cap}\left(\overline{\mathbf{C}} \backslash \Omega_{n}\right) \rightarrow 0
$$

as $n \rightarrow \infty$, it follows from Harnack's theorem that $g_{\Omega_{n}}(z, \infty)-g_{\Omega}(z, \infty)$ tends to zero uniformly on compact subsets of $\Omega$. In particular, these functions are harmonic in $G$, they are uniformly bounded on $G$, they are identically 0 on $\partial \Omega \cap \overline{D_{1}}$ and they tend to zero on compact subsets of $\partial G \backslash \overline{D_{1}}$. Then, by [20, Lemma 7.1] their normal derivatives at $z$ tend to 0 on $\partial \Omega \cap D_{1}$, and this is exactly (3.7). That the convergence is uniform on compact subsets of $\partial \Omega \cap D_{1}$ follows from the proof in 20, Lemma 7.1].

The proof for cases (i) or (iii) is similar.

\section{FAST DECREASING POLYNOMIALS}

We shall need polynomials that take the value 1 at a given (outer) boundary point of a compact set and decrease very fast on the set as we move away from that point. In this section we do this construction.

Theorem 4.1. Let $K$ be a compact subset of the plane, $\Omega$ the unbounded component of its complement, and $Z \in \partial \Omega$ a point on the outer boundary of $K$. Assume that there is a disk in $\Omega$ that contains $Z$ on its boundary. Then for every $\beta>1$ there are constants $d_{\beta}, D_{\beta}>0$, and for every $n=1,2, \ldots$ polynomials $P_{n}$ of degree at most $n$ such that $P_{n}(Z)=1,\left|P_{n}(z)\right| \leq 1$ for $z \in K$ and

$$
\left|P_{n}(z)\right| \leq D_{\beta} e^{-n d_{\beta}|z-Z|^{\beta}}, \quad z \in K .
$$

If $Z$ lies inside a $C^{2}$ boundary open arc $J$ of $\partial \Omega$ such that $J=\partial \Omega \cap O$ with some open set $O$, then the constants $D_{\beta}, d_{\beta}$ for which (4.1) is true for $P_{n}=P_{n, Z, K}$ can be chosen to be independent of $Z$ lying in any closed subarc of $J$.

We also mention that this theorem is optimal in the sense that $\beta=1$ is not possible in it. We shall often use Theorem 4.1 in the following form.

Corollary 4.2. With the assumptions of Theorem 4.1 there are constants $C_{0}, c_{0}>$ 0 , and for every $n$ polynomial $S_{n}=S_{n, Z, K}$ of degree at most $n^{109 / 110}$ such that $S_{n}(Z)=1,\left|S_{n}(z)\right| \leq 1$ for $z \in K$, and for $|z-Z| \geq n^{-9 / 10}, z \in K$, we have

$$
\left|S_{n}(z)\right| \leq C_{0} e^{-c_{0} n^{1 / 110}} .
$$

If $Z$ lies inside a $C^{2}$ boundary open arc $J$ of $\partial \Omega$ such that $J=\partial \Omega \cap O$ with some open set $O$, then the constants $C_{0}, c_{0}$ for which (4.2) is true for $S_{n}=S_{n, Z, K}$ can be chosen to be independent of $Z$ lying in any closed subarc of $J$.

The polynomials $S_{n}$ will be frequently used below. For notational convenience we wrote $n$ in the index, but one should always remember that their degree is at most $n^{109 / 110}=o(n)$. 
Proof. First we prove the theorem for the closed unit disk, thus first let $K=\bar{\Delta}$. It was proved in [12] that for every $\beta>1$ there are constants $c_{\beta}, C_{\beta}>0$ and for every $n=1,2, \ldots$ polynomials $R_{n}$ of degree at most $n$ such that $R_{n}(0)=1,\left|R_{n}(x)\right| \leq 1$ for $x \in[-1,1]$ and

$$
\left|R_{n}(x)\right| \leq C_{\beta} e^{-n c_{\beta}|x|^{\beta}}, \quad x \in[-1,1] .
$$

By replacing $R_{n}(x)$ with $\left(R_{n}(x)+R_{n}(-x)\right) / 2$ if necessary, we may assume that $R_{n}$ is even. Then $R_{n}(\sin (t / 2))$ is a trigonometric polynomial of degree at most $n / 2$. Hence $e^{i[n / 2] t} R_{n}(\sin (t / 2))$ coincides with some $P_{n}^{*}\left(e^{i t}\right)$, where $P_{n}^{*}$ is an algebraic polynomial of degree at most $n$. It is clear from (4.3) that $P_{n}^{*}(1)=1,\left|P_{n}^{*}\left(e^{i t}\right)\right| \leq 1$, and for $t \in[-\pi, \pi]$

$$
\left|P_{n}^{*}\left(e^{i t}\right)\right| \leq C_{\beta} e^{-n c_{\beta}|\sin (t / 2)|^{\beta}} \leq C_{\beta} e^{-n c_{\beta}|t|^{\beta} / \pi^{\beta}},
$$

where we used the fact that $|\sin (t / 2)| \geq|t| / \pi$ for $t \in[-\pi, \pi]$. We claim that this is enough to prove the statement for the unit disk, i.e. $P_{n}^{*}$ also satisfies (4.1). By the maximum principle we certainly have $\left|P_{n}^{*}(z)\right| \leq 1$ in the unit disk.

Let

$$
J_{z}=\left\{e^{i t} \mid t \in[-\pi,-|1-z|] \cup[|1-z|, \pi]\right\}
$$

be the arc of the unit circle consisting of points with arc length distance $\geq|1-z|$ from the point 1 . Let $\omega(z ; J, G)$ denote the harmonic measure of $J \subset \partial G$ at $z$ with respect to a domain $G$. It is clear (apply conformal map onto the upper half plane and note that on the upper half plane the harmonic measure is nothing other (see [2] or [26, Table 4.1]) than $1 / \pi$-times the angle that the set $J$ is seen from the point $z)$ that there is a constant $\alpha$ such that $\omega\left(z ; J_{z}, \Delta\right) \geq \alpha$ for all $z \in \Delta$. Since on $J_{z}$ we have by (4.4) the estimate

$$
\left|P_{n}^{*}\left(e^{i t}\right)\right| \leq C_{\beta} e^{-n c_{\beta}|1-z|^{\beta} / \pi^{\beta}}
$$

and otherwise $\left|P_{n}^{*}(z)\right| \leq 1$ on the complementary boundary arc, it follows from a comparison of the subharmonic function $\log \left|P_{n}^{*}(w)\right|$ with the harmonic function

$$
\omega\left(w ; J_{z}, \Delta\right)\left(\log C_{\beta}-n c_{\beta}|1-z|^{\beta} / \pi^{\beta}\right)
$$

that

$$
\left|P_{n}^{*}(z)\right| \leq C_{\beta}^{\alpha} e^{-n \alpha c_{\beta}|1-z|^{\beta} / \pi^{\beta}}
$$

as was claimed.

After these we complete the proof for arbitrary sets. Since it is assumed that there is a disk in $\Omega$ that contains $Z$ on its boundary, it is easy to construct a simply connected domain $G$ with $C^{2}$ boundary containing $K \backslash Z$ in its interior such that $Z \in \partial G$. Choose a lemniscate $\sigma$ such that $\sigma$ is a Jordan curve, its interior contains $G \backslash Z$ and $Z \in \sigma$. The existence of $\sigma$ immediately follows from Theorem 2.3, Let $T_{N}$ be the polynomial for which $\sigma=\left\{z|| T_{N}(z) \mid=1\right\}$, and without loss of generality we may assume that $T_{N}(Z)=1$. We claim that if $P_{n}^{*}$ are the polynomials from (4.1) for the closed unit disk (see (4.5)), then $P_{n}(z)=P_{[n / N]}^{*}\left(T_{N}(z)\right)$ satisfy (44.1) with some constants. In fact, if $z \in K$ is in a small neighborhood of $Z$, then $T_{N}(z)$ is in a small neighborhood of 1 , and $|z-Z| \sim\left|T_{N}(z)-1\right|$, so (4.1) follows from (4.5) (applied to $T_{N}(z)$ rather than to $z$ ), for, say, $|z-Z| \leq \delta$. On the other hand, if $z \in K$ and $|z-Z| \geq \delta$, then $\left|T_{N}(z)-1\right| \geq \delta_{1}$ for some $\delta_{1}$; hence (4.1) follows again from (4.5). This latter statement regarding $\left|T_{N}(z)-1\right| \geq \delta_{1}$ would not be true for $z$ lying on $\sigma$ since on $\sigma$ there are $N$ points that are mapped by $T_{N}$ into 1 . 
However, since $K$ is contained strictly inside $\sigma$ except for the point $Z$, the existence of $\delta_{1}$ follows.

The uniformity of the constants $D_{\beta}, d_{\beta}$ follows from the construction. In fact, Theorem 2.3 shows that a rotated-translated copy of $\sigma$ can serve as the $\sigma_{Z^{\prime}}$ for all $Z^{\prime}$ lying sufficiently close to $Z$, and by compactness we can infer that the degree of $T_{N}$ that gives $\sigma_{Z}$ can be chosen to be bounded when $Z$ lies in a closed subarc of $J$ (and actually, the $T_{N}$ 's themselves form a compact family). Now the uniformity in question follows from the proof.

Proof of Corollary 4.2. The corollary follows by setting $\beta=12 / 11$ if we apply (4.1) with $n^{109 / 110}$ replacing $n$ and with $|z-Z| \geq n^{-9 / 10}$.

\section{Proof of Theorems 1.3 and 1.4}

In what follows $\Delta_{\delta}(z)=\{w|| w-z \mid<\delta\}$ is the disk of radius $\delta$ with its center at $z$.

First we shall prove Theorem 1.4 for lemniscate domains, and those will be general enough to yield the full theorem. For simpler notation, if $W$ is a weight function and $d \mu=W d A$, then we shall write $\lambda_{n}(W, z)$ instead of $\lambda_{n}(\mu, z)=\lambda_{n}(W d A, z)$.

5.1. Proof of Theorem 1.3 for the unit disk and identically 1 weight. We need the special case of Theorem 1.4 for the unit disk $K=\bar{\Delta}$, resp. for the $\operatorname{ring} K=\bar{\Delta}_{\rho}^{*}=\{1-\rho \leq|z| \leq 1\}$ and for $W \equiv 1$. Since in this case we have circular symmetry, we shall consider the Christoffel functions at $z_{0}=1$. Thus, let $Q_{n}(w)=\sum_{j=1}^{n} a_{j} w^{j}$ be a polynomial of degree at most $n$ with $Q_{n}(1)=1$. Then $\sum_{j=1}^{n} a_{j}=1$, and

$$
\begin{aligned}
\int_{\bar{\Delta}}\left|Q_{n}(w)\right|^{2} d A(w) & =\int_{0}^{1} r \int_{0}^{2 \pi}\left|Q_{n}\left(r e^{i t}\right)\right|^{2} d t d r=2 \pi \int_{0}^{1}\left(\sum_{j=0}^{n}\left|a_{j}\right|^{2} r^{2 j+1}\right) d r \\
& =2 \pi \sum_{j=0}^{n} \frac{\left|a_{j}\right|^{2}}{2 j+2} .
\end{aligned}
$$

But by the Cauchy-Schwarz inequality

$$
\begin{aligned}
1=\sum_{j=0}^{n} a_{n} & \leq \sum_{j=0}^{n}\left|a_{j}\right| \leq\left(\sum_{j=0}^{n} \frac{\left|a_{j}\right|^{2}}{2 j+2}\right)^{1 / 2}\left(\sum_{j=0}^{n}(2 j+2)\right)^{1 / 2} \\
& =\left(\sum_{j} \frac{\left|a_{j}\right|^{2}}{2 j+2}\right)^{1 / 2}((n+1)(n+2))^{1 / 2}
\end{aligned}
$$

i.e.

$$
\sum_{j=0}^{n} \frac{\left|a_{j}\right|^{2}}{2 j+2} \geq \frac{1}{(n+1)(n+2)},
$$

and we have equality if $a_{j}=(2 j+2) /(n+1)(n+2)$, i.e. for

$$
Q_{n}(w)=\sum_{j=0}^{n} \frac{(2 j+2) w^{j}}{(n+1)(n+2)} .
$$


These give

$$
\lambda_{n}\left(\left.1\right|_{\bar{\Delta}}, 1\right)=\frac{2 \pi}{(n+1)(n+2)}=(1+o(1)) \frac{2 \pi}{n^{2}},
$$

where $\left.{ }^{1}\right|_{\bar{\Delta}}$ is the weight that is identically 1 on $\bar{\Delta}$. Here, and everywhere below, $o$ (1) denotes a quantity that tends to 0 as $n$ (or $m$ if that is the running index) tends to $\infty$.

In a similar manner, if $\left.1\right|_{\Delta_{\rho}^{*}}$ is the weight that is identically 1 on the ring $\overline{\Delta_{\rho}^{*}}=\{\rho \leq|z| \leq 1\}$, then the above calculation gives

$$
\int_{\overline{\Delta_{\rho}^{*}}}\left|Q_{n}(w)\right|^{2} d A(w)=2 \pi \sum_{j=0}^{n}\left|a_{j}\right|^{2} \frac{1-\rho^{2 j+2}}{2 j+2},
$$

and since

$$
\sum_{j=0}^{n} \frac{2 j+2}{1-\rho^{2 j+2}}=(1+o(1)) n^{2},
$$

we have, as before,

$$
\lambda_{n}\left(\left.1\right|_{\Delta_{\rho}^{*}}, 1\right)=(1+o(1)) \frac{2 \pi}{n^{2}} .
$$

5.2. Proof of Theorem 1.3 for lemniscate domains and for positive weights. In this subsection we prove Theorem 1.4 for lemniscate domains $K=L$ and for weights $W$ that are strictly positive on the boundary $\partial L$.

Thus, let $L=T_{N}^{-1}(\bar{\Delta})$ be as explained in Section 2, We keep the notation from there; see in particular the definitions of the sets $G_{i}$. Assume that $z_{0} \in \partial G_{1}$ and $T_{N}\left(z_{0}\right)=1$.

First we prove the lower estimate for the special weight $W_{0}$ that is defined to be $W_{0}(z)=\left|T_{N}^{\prime}(z)\right|^{2}$ for $z \in \overline{L_{\rho}^{*}}$ and $W_{0}(z)=0$ otherwise (i.e. on the set $\tilde{L}_{\rho}$, which is the inverse image of $\{|z|<\rho\}$, we set $W_{0}$ to be 0$)$. Let $P_{m}$ be an optimal polynomial for $\lambda_{m}\left(W_{0}, z_{0}\right)$, i.e. $P_{m}\left(z_{0}\right)=1$ and

$$
\int_{L_{\rho}^{*}}\left|P_{m}(z)\right|^{2}\left|T_{N}^{\prime}(z)\right|^{2} d A(z)=\lambda_{m}\left(W_{0}, z_{0}\right)
$$

As in Section 2, for $z \in L_{\rho}^{*}$ let $z_{j}, j=1, \ldots, N$, be the $N$ distinct points with $T_{N}\left(z_{j}\right)=T_{N}(z)$, and with the polynomials $S_{m}=S_{m, z_{0}, L}$ from Corollary 4.2 (which are of degree at most $m^{109 / 110}$ ) consider the polynomial

$$
R_{m}(z)=P_{m}(z) S_{m}(z) \prod_{j=2}^{N} \frac{z-\left(z_{0}\right)_{j}}{z_{0}-\left(z_{0}\right)_{j}}
$$

of degree at most $m+m^{109 / 110}+N$. This satisfies $R_{m}\left(z_{0}\right)=1, R_{m}\left(\left(z_{0}\right)_{j}\right)=0$ for all $j=2, \ldots, m$,

$$
\left|R_{m}(z)\right| \leq(1+o(1))\left|P_{m}(z)\right|, \quad \text { for } z \in G_{1},
$$

and for all $i=2, \ldots, N$ we have with $\eta_{m}=C_{0} \exp \left(-\left(c_{0} / 2\right) m^{1 / 110}\right)$ (see Corollary 4.2) $\left|R_{m}(z)\right| \leq \eta_{m}\left|P_{m}(z)\right|$ for all large $m$ (we write $c_{0} / 2$ in the definition of $\eta_{m}$ 
instead $c_{0}$ as in Corollary 4.2 in order to take care of the third polynomial on the right of (5.4)). Hence, from (2.7) it follows that for $i \neq 1$ and all $j$

$$
\begin{aligned}
\int_{G_{j}}\left|R_{m}\left(z_{i}\right)\right|^{2}\left|T_{N}^{\prime}\left(z_{j}\right)\right|^{2} d A\left(z_{j}\right) & =\int_{G_{i}}\left|R_{m}\left(z_{i}\right)\right|^{2}\left|T_{N}^{\prime}\left(z_{i}\right)\right|^{2} d A\left(z_{i}\right) \\
& \leq \eta_{m} \int\left|P_{m}(z)\right|^{2} W_{0}(z) d A(z) \\
& =\eta_{m} \lambda_{m}\left(W_{0}, z_{0}\right)
\end{aligned}
$$

while

$$
\begin{aligned}
\int_{G_{j}}\left|R_{m}\left(z_{1}\right)\right|^{2}\left|T_{N}^{\prime}\left(z_{j}\right)\right|^{2} d A\left(z_{j}\right) & =\int_{G_{1}}\left|R_{m}\left(z_{1}\right)\right|^{2}\left|T_{N}^{\prime}\left(z_{1}\right)\right|^{2} d A\left(z_{1}\right) \\
& \leq(1+o(1)) \int\left|P_{m}(z)\right|^{2} W_{0}(z) d A(z) \\
& =(1+o(1)) \lambda_{m}\left(W_{0}, z_{0}\right) .
\end{aligned}
$$

Hence, by Hölder's inequality,

$$
\begin{aligned}
\int_{G_{j}}\left|R_{m}\left(z_{i}\right)\right|\left|R_{m}\left(z_{k}\right)\right|\left|T_{N}^{\prime}\left(z_{j}\right)\right|^{2} d A\left(z_{j}\right) & \leq \sqrt{(1+o(1)) \lambda_{m}\left(W_{0}, z_{0}\right)} \sqrt{\eta_{m} \lambda_{m}\left(W_{0}, z_{0}\right)} \\
& =(1+o(1)) \sqrt{\eta_{m}} \lambda_{m}\left(W_{0}, z_{0}\right)
\end{aligned}
$$

except when $i=k=1$, in which case the left hand side is the same as in (5.6). These give

$$
\begin{aligned}
\int_{G_{j}}\left|\sum_{i=1}^{N} R_{m}\left(z_{i}\right)\right|^{2}\left|T_{N}^{\prime}\left(z_{j}\right)\right|^{2} d A\left(z_{j}\right) & \leq(1+o(1))\left(\lambda_{m}\left(W_{0}, z_{0}\right)+N^{2} \sqrt{\eta_{m}} \lambda_{m}\left(W_{0}, z_{0}\right)\right) \\
& =(1+o(1)) \lambda_{m}\left(W_{0}, z_{0}\right),
\end{aligned}
$$

and so

$$
\int_{L_{\rho}^{*}}\left|\sum_{i=1}^{N} R_{m}\left(z_{i}\right)\right|^{2}\left|T_{N}^{\prime}\left(z_{j}\right)\right|^{2} d A\left(z_{j}\right)=\sum_{j=1}^{N} \int_{G_{j}} \leq N(1+o(1)) \lambda_{m}\left(W_{0}, z_{0}\right) .
$$

Now we utilize an idea from [37. The expression $\sum_{i=1}^{N} R_{m}\left(z_{i}\right)$ is a symmetric polynomial in the zeros of the equation $T_{N}(t)=T_{N}(z)$ (in the equation $t$ is the unknown). Hence it can be expressed as a polynomial of the elementary symmetric polynomials in $z_{1}, \ldots, z_{n}$ and hence (by Viète's formulae) as a polynomial in the coefficients of $T_{N}(t)-T_{N}(z)$. Thus,

$$
\sum_{i=1}^{N} R_{m}\left(z_{i}\right)=Q_{m}\left(T_{N}(z)\right)
$$

with some polynomial $Q_{m}$ of degree at most

$$
\operatorname{deg}\left(R_{m}\right) / N=\left(m+m^{109 / 110}+N\right) / N
$$

(as for the degree of $R_{m}$, consider that for any $k$, if we write $z_{1}^{k}+\cdots+z_{N}^{k}$ in terms of elementary symmetric polynomials, then the expression has to be homogeneous of degree $k$; hence the degree of

$$
z_{1} z_{2} \cdots z_{N}=(-1)^{N}\left(T_{N}(0)-T_{N}(z)\right)
$$

in the expression is at most $k / N)$. 
Now (5.7), in conjunction with (2.4) (more precisely, with its analogue for $L_{\rho}^{*}$ ), yields

$$
\int_{\overline{\Delta_{\rho}^{*}}}\left|Q_{m}(w)\right|^{2} d A(w) \leq(1+o(1)) \lambda_{m}\left(W_{0}, z_{0}\right) .
$$

Since

$$
Q_{m}(1)=Q_{m}\left(T_{N}\left(z_{0}\right)\right)=\sum_{j=1}^{N} R_{m}\left(\left(z_{0}\right)_{j}\right)=1,
$$

it follows from (5.3) that the left hand side in the preceding inequality is at least

$$
(1+o(1)) \frac{2 \pi}{\left(\operatorname{deg}\left(Q_{m}\right)\right)^{2}} \geq(1+o(1)) \frac{2 \pi N^{2}}{m^{2}} .
$$

Thus, finally we obtain

$$
\lambda_{m}\left(W_{0}, z_{0}\right) \geq(1+o(1)) \frac{2 \pi N^{2}}{m^{2}} .
$$

Next we prove the matching upper estimate, but for the weight that is identically 1 on all of $L$ (recall that $W_{0}$ was 0 on the inner domain $\tilde{L}_{\rho}$ ). This time let $Q_{n}$ be the polynomial defined in (5.1) for which $Q_{n}(1)=1$ and

$$
\int_{\bar{\Delta}}\left|Q_{n}(w)\right|^{2} d A(w)=\lambda_{n}\left(\left.1\right|_{\bar{\Delta}}, 1\right)
$$

Set $R_{n}(z)=Q_{n}\left(T_{N}(z)\right)$, which is a polynomial of degree $n N$ with $R_{n}\left(z_{0}\right)=1$ and $($ see $(2.5))$

$$
\int_{G_{j}}\left|R_{n}(z)\right|^{2}\left|T_{N}^{\prime}(z)\right|^{2} d A(z)=\int_{\Delta_{\rho}^{*}}\left|Q_{n}(w)\right|^{2} d A(w) \leq \lambda_{n}\left(\left.1\right|_{\bar{\Delta}}, 1\right)
$$

for all $j$, while by (2.4)

$$
\int_{\tilde{L}_{\rho}}\left|R_{n}(z)\right|^{2}\left|T_{N}^{\prime}(z)\right|^{2} d A(z)=N \int_{\Delta_{\rho}}\left|Q_{n}(w)\right|^{2} d A(w) \leq N \lambda_{n}\left(\left.1\right|_{\bar{\Delta}}, 1\right),
$$

where, as in (2.3), $\tilde{L}_{\rho}=T_{N}^{-1}\left(\Delta_{\rho}\right)=T_{N}^{-1}\{|w| \leq \rho\}$ is the inner region surrounded by the $G_{j}$ 's. If we multiply $R_{n}$ by the polynomial $S_{n}=S_{n, z_{0}, L}$ from Corollary 4.2 , then the previous formulae give with $\eta_{n}=C_{0} \exp \left(-\left(c_{0} / 2\right) n^{1 / 110}\right)$

$$
\int_{G_{j}}\left|R_{n}(z) S_{n}(z)\right|^{2}\left|T_{N}^{\prime}(z)\right|^{2} d A(z) \leq \eta_{n} \lambda_{n}\left(\left.1\right|_{\bar{\Delta}}, 1\right),
$$

for all $j=2, \ldots, N$,

$$
\int_{G_{1}}\left|R_{n}(z) S_{n}(z)\right|^{2}\left|T_{N}^{\prime}(z)\right|^{2} d A(z) \leq \int_{G_{1}}\left|R_{n}(z)\right|^{2}\left|T_{N}^{\prime}(z)\right|^{2} d A(z) \leq \lambda_{n}\left(\left.1\right|_{\bar{\Delta}}, 1\right),
$$

and

$$
\int_{\tilde{L}_{\rho}}\left|R_{n}(z) S_{n}(z)\right|^{2}\left|T_{N}^{\prime}(z)\right|^{2} d A(z) \leq \eta_{n} N \lambda_{n}\left(\left.1\right|_{\bar{\Delta}}, 1\right) .
$$

Note now that $R_{n}(z) S_{n}(z) T_{N}^{\prime}(z) / T_{N}^{\prime}\left(z_{0}\right)$ is a polynomial of degree at most $n N+$ $n^{109 / 110}+N-1$ that takes the value 1 at $z_{0}$. Hence the preceding inequalities when we sum them up give for $m \geq n N+n^{109 / 110}+N-1 \geq \operatorname{deg}\left(R_{n} S_{n} T_{N}^{\prime}\right)$

$$
\lambda_{m}\left(\left.1\right|_{L}, z_{0}\right) \leq(1+o(1)) \frac{1}{\left|T_{N}^{\prime}\left(z_{0}\right)\right|^{2}} \lambda_{n}\left(\left.1\right|_{\bar{\Delta}}, 1\right) .
$$


For a given $m$ select the largest $n$ with $m \geq n N+n^{109 / 110}+N-1$, and then we can infer from (5.2) the inequality

$$
\lambda_{m}\left(\left.1\right|_{L}, z_{0}\right) \leq(1+o(1)) \frac{N^{2}}{\left|T_{N}^{\prime}\left(z_{0}\right)\right|^{2}} \frac{2 \pi}{m^{2}} .
$$

Now it is easy to complete the proof for lemniscates and for weights that are strictly positive on the boundary. Indeed, this is the same as replacing the weight $W_{0}$ in (5.8) and the weight $\left.1\right|_{L}$ in (5.9) by an arbitrary continuous weight that is positive on (and hence around) the boundary. In fact, first let $W\left(z_{0}\right)>0$ and let $P_{m}$ be a polynomial that verifies (5.9), i.e. $P_{m}\left(z_{0}\right)=1$ and

$$
\int_{L}\left|P_{m}(z)\right|^{2} d A(z) \leq(1+o(1)) \frac{N^{2}}{\left|T_{N}^{\prime}\left(z_{0}\right)\right|^{2}} \frac{2 \pi}{m^{2}} .
$$

Since for the polynomials $S_{m}$ from (4.2) we have $W(z)\left|S_{m}(z)\right|^{2} \leq(1+o(1)) W\left(z_{0}\right)$, we get (note that the degree of $S_{m}$ is at most $m^{109 / 110}$ )

$$
\begin{aligned}
\lambda_{m+m^{109 / 110}}\left(W, z_{0}\right) & \leq \int_{L}\left|P_{m}(z) S_{m}(z)\right|^{2} W(z) d A(z) \\
& \leq(1+o(1)) W\left(z_{0}\right) \int_{L}\left|P_{m}(z)\right|^{2} d A(z) \\
& \leq(1+o(1)) W\left(z_{0}\right) \frac{N^{2}}{\left|T_{N}^{\prime}\left(z_{0}\right)\right|^{2}} \frac{2 \pi}{m^{2}}
\end{aligned}
$$

(see (5.9) ), and hence

$$
\limsup _{m \rightarrow \infty} m^{2} \lambda_{m}\left(W, z_{0}\right) \leq 2 \pi W\left(z_{0}\right) \frac{N^{2}}{\left|T_{N}^{\prime}\left(z_{0}\right)\right|^{2}} .
$$

This proof used only $W\left(z_{0}\right)>0$.

We have to show a matching lower bound for the case when $W$ is strictly positive in $L_{\rho}^{*}$ for some $\rho<1$ (see the definition (2.3) ). Then

$$
\frac{W\left(z_{0}\right)}{\left|T_{N}^{\prime}\left(z_{0}\right)\right|^{2}}\left|T_{N}^{\prime}(z)\right|^{2}\left|S_{n}(z)\right|^{2} \leq(1+o(1)) W(z)
$$

uniformly on $\overline{L_{\rho}^{*}}$, so if $P_{m}$ are the polynomials that are optimal for $\lambda_{m}\left(W, z_{0}\right)$, i.e. $P_{m}\left(z_{0}\right)=1$, and

$$
\int_{L}\left|P_{m}(z)\right|^{2} W(z) d A(z)=\lambda_{m}\left(W, z_{0}\right)
$$

then

$$
\begin{array}{ll}
\frac{W\left(z_{0}\right)}{\left|T_{N}^{\prime}\left(z_{0}\right)\right|^{2}} \int_{L_{\rho}^{*}}\left|P_{m}(z) S_{m}(z)\right|^{2}\left|T_{N}^{\prime}(z)\right|^{2} d A(z) & \leq(1+o(1)) \int_{L}\left|P_{m}(z)\right|^{2} W(z) d A(z) \\
(5.11) & =(1+o(1)) \lambda_{m}\left(W, z_{0}\right) .
\end{array}
$$

Note that since $P_{m}\left(z_{0}\right) S_{m}\left(z_{0}\right)=1$, the left hand side is at least

$$
\frac{W\left(z_{0}\right)}{\left|T_{N}^{\prime}\left(z_{0}\right)\right|^{2}} \lambda_{m+m^{109 / 110}}\left(W_{0}, Z_{0}\right)
$$

hence (5.8) yields

$$
\lambda_{m}\left(W, z_{0}\right) \geq(1+o(1)) W\left(z_{0}\right) \frac{N^{2}}{\left|T_{N}^{\prime}\left(z_{0}\right)\right|^{2}} \frac{2 \pi}{m^{2}},
$$


i.e.

$$
\liminf _{m \rightarrow \infty} m^{2} \lambda_{m}\left(W, z_{0}\right) \geq 2 \pi W\left(z_{0}\right) \frac{N^{2}}{\left|T_{N}^{\prime}\left(z_{0}\right)\right|^{2}} .
$$

This is derived under the assumption $W>0$ on some $L_{\rho}^{*}$. In view of (2.2) formulas (5.10) and (5.12) constitute a proof of Theorem 1.4 for the lemniscate set $L$ and for weights $W$ that are strictly positive on $\partial L$.

5.3. Proof of Theorem $\mathbf{1 . 3}$ in its full generality. First we prove a lower estimate on $\lambda_{n}\left(W, z_{0}\right)$ under the condition $W\left(z_{0}\right)>0$. Thus, let $z_{0} \in \partial \Omega, W\left(z_{0}\right)>0$, and assume, as in the theorem, that $\partial K$ is a $C^{2}$ Jordan arc in a neighborhood of $z_{0}$, and with $U=\{z \mid W(z)>0\} \cap \operatorname{Int}(K)$ we have $\operatorname{cap}(U)=\operatorname{cap}(K)$. We choose a small $\delta$ such that $\Delta_{\delta}\left(z_{0}\right) \cap \partial K$ is a $C^{2}$ arc and $W>0$ in $\overline{\Delta_{\delta}\left(z_{0}\right)} \cap \partial K$.

For $k=1,2, \ldots$ let $U_{k}^{*}=\{z \mid W(z)>1 / k\} \cap \operatorname{Int}(K)$, and let $U_{k}$ be the union of all those connected components of $U_{k}^{*}$ which contain a ball of radius $\geq 1 / k$. Then $U_{k}$ has finitely many components, $U_{k} \subseteq U_{k+1}, \bigcup_{k} U_{k}=U$, hence cap $\left(U_{k}\right) \rightarrow$ $\operatorname{cap}(U)=\operatorname{cap}(K)$. Also, since $W>0$ in $\overline{\Delta_{\delta}\left(z_{0}\right)} \cap K$ and $W$ is continuous, we have $\overline{\Delta_{\delta}\left(z_{0}\right)} \cap K \subseteq \overline{U_{k}}$ for all sufficiently large $k$. For each $l=1,2, \ldots$ consider the dyadic squares

$$
\left\{(x, y) \mid u 2^{-l} \leq x \leq(u+1) 2^{-l}, v 2^{-l} \leq y \leq(v+1) 2^{-l}\right\},
$$

where $u, v$ run through the integers, and let $V_{k}^{l}$ be the union of those dyadic squares that lie inside $U_{k}$ together with the eight neighboring dyadic squares. Then $V_{k}^{l}$ is closed, its boundary consists of finitely many curves (indeed, broken lines, but they may not be simple, i.e. they may have self-intersection) and any two components of $V_{k}$ are of distance $\geq 1 / 2^{l}$ (note that two dyadic squares either intersect or their distance is $\left.\geq 1 / 2^{l}\right)$. Therefore, if $U_{k}^{l}$ is the $1 /\left(3 \cdot 2^{l}\right)$-neighborhood of $V_{k}^{l}$, then $U_{k}^{l}$ is an open subset of $U_{k}$ such that its boundary consists of finitely many Jordan curves. Easy inspection shows that $U_{k}^{l} \subseteq U_{k}^{l+1}, \bigcup_{l} U_{k}^{l}=U_{k}$, and hence cap $\left(U_{k}^{l}\right) \rightarrow \operatorname{cap}\left(U_{k}\right)$ as $l \rightarrow \infty$. Select an increasing sequence $\left\{l_{k}\right\}$ such that $\operatorname{cap}\left(U_{k}^{l_{k}}\right) \rightarrow \operatorname{cap}(K)$ (this is possible since $\left.\operatorname{cap}\left(U_{k}\right) \rightarrow \operatorname{cap}(K)\right)$, and set

$$
H_{k}=\overline{U_{k}^{l_{k}} \cup\left(\Delta_{\delta}\left(z_{0}\right) \cap \operatorname{Int}(K)\right)} .
$$

This is a closed set, and for large $k$ its boundary consists of finitely many Jordan curves. Furthermore, $H_{k} \subseteq H_{k+1}$ for all $k$ and $\operatorname{cap}\left(H_{k}\right) \rightarrow \operatorname{cap}(K)$ as $k \rightarrow \infty$. Note that in a neighborhood of $z_{0}$ the boundaries of $H_{k}$ and $K$ are the same, and hence we can conclude from Lemma 3.3 for the corresponding normal derivatives the convergence

$$
\frac{\partial g_{\overline{\mathbf{C}} \backslash H_{k}}\left(z_{0}, \infty\right)}{\partial \mathbf{n}} \rightarrow \frac{\partial g_{\overline{\mathbf{C}} \backslash K}\left(z_{0}, \infty\right)}{\partial \mathbf{n}}
$$

as $k \rightarrow \infty$. For given $\varepsilon>0$ fix $k$ so that

$$
\frac{\partial g_{\overline{\mathbf{C}} \backslash H_{k}}\left(z_{0}, \infty\right)}{\partial \mathbf{n}} \leq \frac{\partial g_{\overline{\mathbf{C}} \backslash K}\left(z_{0}, \infty\right)}{\partial \mathbf{n}}+\varepsilon .
$$

By Theorem 2.2 (see the last sentence in it) there is a lemniscate $\sigma=T^{-1}(\mathcal{C})$ such that $\sigma \subset H_{k}, z_{0} \in \sigma$ and if $L=T^{-1}(\Delta)$ denotes the lemniscate domain enclosed by $\sigma$, then

$$
\frac{\partial g_{\overline{\mathbf{C} \backslash L}}\left(z_{0}, \infty\right)}{\partial \mathbf{n}} \leq \frac{\partial g_{\overline{\mathbf{C}} \backslash H_{k}}(z, \infty)}{\partial \mathbf{n}}+\varepsilon .
$$


Note that $L$ may not be part of $H_{k}$, since the components of $\operatorname{Int}\left(H_{k}\right)$ may not be simply connected. However, since $\sigma \subset H_{k} \subset U_{k}$, the weight $W$ is strictly positive $(\geq 1 / k)$ on $\sigma$. Therefore we can apply (5.12) to $L$ to conclude

$$
\begin{aligned}
n^{2} \lambda_{n}\left(W, z_{0}\right) & \geq n^{2} \lambda_{n}\left(\left.W\right|_{L \cap H_{k}}, z_{0}\right) \\
& \geq(1+o(1)) 2 \pi W\left(z_{0}\right)\left(\frac{\partial g_{\overline{\mathbf{C}} \backslash L}\left(z_{0}, \infty\right)}{\partial \mathbf{n}}\right)^{-2} \\
& \geq(1+o(1)) 2 \pi W\left(z_{0}\right)\left(\frac{\partial g_{\overline{\mathbf{C}} \backslash K}\left(z_{0}, \infty\right)}{\partial \mathbf{n}}+2 \varepsilon\right)^{-2} .
\end{aligned}
$$

For $\varepsilon \rightarrow 0$ this yields

$$
\liminf _{n \rightarrow \infty} n^{2} \lambda_{n}\left(W, z_{0}\right) \geq 2 \pi W\left(z_{0}\right)\left(\frac{\partial g_{\overline{\mathbf{C}} \backslash K}\left(z_{0}, \infty\right)}{\partial \mathbf{n}}\right)^{-2} .
$$

Note that (5.16) was derived under the assumption $W\left(z_{0}\right)>0$.

The matching upper estimate on $\lambda_{n}(W)$ is obtained in a similar manner. Since we are now establishing an upper bound, we can work with $W(z)+\varepsilon$ rather than $W(z)$. Let $K \subset K_{k}$ be such that $\operatorname{Int}\left(K_{k}\right)$ is the union of finitely many Jordan domains, $K_{k+1} \subseteq K_{k}, \cap_{k} K_{k}=K$, with some small $\delta>0$ we have $\overline{\Delta_{\delta}\left(z_{0}\right)} \cap K_{k}=\overline{\Delta_{\delta}\left(z_{0}\right)} \cap K$ for all $k$, and $\overline{\Delta_{\delta}\left(z_{0}\right)} \cap \partial K$ (which is a $C^{2}$ curve) lies on the outer boundary of each $K_{k}$. It is easy to construct such a family. Lemma 3.3 gives

$$
\frac{\partial g_{\overline{\mathbf{C}} \backslash K_{k}}\left(z_{0}, \infty\right)}{\partial \mathbf{n}} \rightarrow \frac{\partial g_{\overline{\mathbf{C}} \backslash K}\left(z_{0}, \infty\right)}{\partial \mathbf{n}} \quad \text { as } k \rightarrow \infty .
$$

For given $\varepsilon>0$ fix $k$ so that

$$
\frac{\partial g_{\overline{\mathbf{C} \backslash K_{k}}}\left(z_{0}, \infty\right)}{\partial \mathbf{n}} \geq \frac{\partial g_{\overline{\mathbf{C} \backslash K}}\left(z_{0}, \infty\right)}{\partial \mathbf{n}}-\varepsilon .
$$

According to Theorem 2.3 there is a closed lemniscate domain $L=T^{-1}(\bar{\Delta})$ such that $K_{k} \subset L, z_{0} \in \partial L$ and

$$
\frac{\partial g_{\overline{\mathbf{C} \backslash L}}\left(z_{0}, \infty\right)}{\partial \mathbf{n}} \geq \frac{\partial g_{\overline{\mathbf{C}} \backslash K_{k}}(z, \infty)}{\partial \mathbf{n}}-\varepsilon .
$$

Extend $W+\varepsilon$ to a strictly positive continuous function $W^{*}$ on $L$. We can apply (5.10) to $L$ to conclude

$$
\begin{aligned}
& n^{2} \lambda_{n}\left(W, z_{0}\right) \leq n^{2} \lambda_{n}\left(W^{*}, z_{0}\right) \leq(1+o(1)) 2 \pi W^{*}\left(z_{0}\right)\left(\frac{\partial g_{\overline{\mathbf{C}} \backslash L}\left(z_{0}, \infty\right)}{\partial \mathbf{n}}\right)^{-2} \\
& \leq(1+o(1)) 2 \pi\left(W\left(z_{0}\right)+\varepsilon\right)\left(\frac{\partial g_{\overline{\mathbf{C}} \backslash K}\left(z_{0}, \infty\right)}{\partial \mathbf{n}}-2 \varepsilon\right)^{-2},
\end{aligned}
$$

where we used that $W^{*}\left(z_{0}\right)=W\left(z_{0}\right)+\varepsilon$. Now for $\varepsilon \rightarrow 0$ this yields

$$
\limsup _{n \rightarrow \infty} n^{2} \lambda_{n}\left(W, z_{0}\right) \leq 2 \pi W\left(z_{0}\right)\left(\frac{\partial g_{\overline{\mathbf{C}} \backslash K}\left(z_{0}, \infty\right)}{\partial \mathbf{n}}\right)^{-2} .
$$

Note that in this derivation we did not use $W\left(z_{0}\right)>0$. 
Finally, (5.16) and (5.20) prove the theorem when $W\left(z_{0}\right)>0$. When $W\left(z_{0}\right)=0$, the theorem follows from (5.20).

5.4. Proof of Theorem 1.4. We can be brief, for most of the time we just follow the preceding proof. We keep the notation from the preceding subsections.

Upper estimate. First of all, the analogue of (5.10) (for lemniscates) is

$$
\limsup _{m \rightarrow \infty} m^{2} \lambda_{m}\left(\mu, z_{0}\right) \leq 2 \pi W\left(z_{0}\right) \frac{N^{2}}{\left|T_{N}^{\prime}\left(z_{0}\right)\right|^{2}} .
$$

This is true, because the $\left|P_{m}\right|$ in the proof of (5.10) attained its maximum value $(=1)$ at $z_{0}$; hence $P_{m} S_{m}$ is very small $\left(\leq C_{0} \exp \left(-c_{0} m^{1 / 110}\right)\right)$ on $L$ outside any neighborhood $U$ of $z_{0}$ for all large $n$. Therefore, the formula before (5.10) now takes the form

$$
\begin{aligned}
\lambda_{m+m^{109 / 110}}\left(\mu, z_{0}\right) \leq & \int_{L \cap U}\left|P_{m}(z) S_{m}(z)\right|^{2} W(z) d A(z) \\
& \quad+\int_{L \backslash U}\left|P_{m}(z) S_{m}(z)\right|^{2} d \mu(z) \\
\leq & (1+o(1)) W\left(z_{0}\right) \int_{L \cap U}\left|P_{m}(z)\right|^{2} d A(z)+C e^{-c_{0} m^{1 / 110}} \\
\leq & (1+o(1)) W\left(z_{0}\right) \frac{N^{2}}{\left|T_{N}^{\prime}\left(z_{0}\right)\right|^{2}} \frac{2 \pi}{m^{2}}
\end{aligned}
$$

and so (5.21) follows. Note that this works for any finite measure $\mu$ which is of the form $W(z) d A(z)$ in a neighborhood of $z_{0}$ with a $W$ that is continuous at $z_{0}$.

Now we can repeat the reasoning of (5.17) $-(5.20)$ to conclude

$$
\limsup _{n \rightarrow \infty} n^{2} \lambda_{n}\left(\mu, z_{0}\right) \leq 2 \pi W\left(z_{0}\right)\left(\frac{\partial g_{\overline{\mathbf{C} \backslash K}}\left(z_{0}, \infty\right)}{\partial \mathbf{n}}\right)^{-2}
$$

exactly as there.

Lower estimate. Let $P_{m}$ be an optimal polynomial for $\lambda_{m}\left(\mu, z_{0}\right)$. $\mu$ being in the Reg class implies (see 33. Thorem 3.2.3]) that if $\left\{Q_{m}\right\}$ is a sequence of polynomials of corresponding degree $m=1,2, \ldots$ and if

$$
\limsup _{m \rightarrow \infty}\left\|Q_{m}\right\|_{L^{2}(\mu)}^{1 / m} \leq 1
$$

then

$$
\limsup _{m \rightarrow \infty}\left\|Q_{m}\right\|_{K}^{1 / m} \leq 1
$$

where the norm $\|\cdot\|_{K}$ on the left hand side is the supremum norm on $K$. In particular, for $P_{m}$ we get the relation

$$
\left\|P_{m}\right\|_{K} \leq e^{\varepsilon_{m} m}
$$

with some $\varepsilon_{m} \rightarrow 0$. We may assume $\varepsilon_{m} \geq 1 / \sqrt{m}$.

As in (5.13) - (5.15), for given $\varepsilon>0$ we can find a lemniscate $\sigma=T_{N}^{-1}(\mathcal{C})$ such that $\sigma$ lies inside $\operatorname{Pc}(K)$ except for the point $z_{0}$ which belongs to $\sigma$ (hence $\sigma$ touches the boundary of $K$ at $\left.z_{0}\right)$, and with $L=T_{N}^{-1}(\bar{\Delta})=\operatorname{Pc}(\sigma)$

$$
\frac{\partial g_{\overline{\mathbf{C} \backslash L}}\left(z_{0}, \infty\right)}{\partial \mathbf{n}} \leq \frac{\partial g_{\overline{\mathbf{C} \backslash K}}(z, \infty)}{\partial \mathbf{n}}+\varepsilon .
$$


Indeed, for this we can copy the argument before (5.13) relying on the condition $\operatorname{cap}(K)=\operatorname{cap}(\operatorname{Int}(\operatorname{Pc}(K)))$ (set there in that proof $W \equiv 1$ on $\operatorname{Pc}(K))$. Let $U=$ $\Delta_{\delta}\left(z_{0}\right)$ be a neighborhood of $z_{0}$ such that in $U \cap K$ we have $d \mu(z)=W(z) d A(z)$ with a $W$ that is continuous at $z_{0}$. We may also assume $W\left(z_{0}\right)>0$, for otherwise the trivial lower bound 0 suffices. We may then assume $\delta$ so small that $W\left(z_{0}\right) / 2 \leq$ $W(z) \leq 2 W\left(z_{0}\right)$ for $z \in U \cap K$.

Choose $\beta=3 / 2, Z=z_{0}$ in Theorem 4.1 consider the polynomials in that theorem of degree $m \varepsilon_{m}^{1 / 2}$, and denote them by $H_{m}$; i.e. $H_{m}$ is of degree at most $m \varepsilon_{m}^{1 / 2}, H_{m}\left(z_{0}\right)=1,\left|H_{m}(z)\right| \leq 1$ for $z \in K$ and

$$
\left|H_{m}(z)\right| \leq D_{\beta} e^{-\left(m \varepsilon_{m}^{1 / 2}\right) d_{\beta}\left|z-z_{0}\right|^{\beta}} .
$$

Then for $z \notin U, z \in \operatorname{Pc}(K)$ we have (see (5.23))

$$
\begin{aligned}
\left|P_{m}(z) H_{m}(z)\right| & \leq D_{\beta} \exp \left(\varepsilon_{m} m-m \varepsilon_{m}^{1 / 2} d_{\beta}\left|z-z_{0}\right|^{\beta}\right) \\
& \leq D_{\beta} \exp \left(\varepsilon_{m} m-m \varepsilon_{m}^{1 / 2} d_{\beta} \delta^{\beta}\right)<\exp \left(-m^{1 / 2}\right)
\end{aligned}
$$

for all large $m$, where we used the fact that $\varepsilon_{m} \geq 1 / \sqrt{m}$.

Now instead of (5.11) we only state the weaker

$$
\begin{array}{ll}
\frac{W\left(z_{0}\right)}{\left|T_{N}^{\prime}\left(z_{0}\right)\right|^{2}} \int_{L_{\rho}^{*} \cap U}\left|P_{m}(z) S_{m}(z)\right|^{2}\left|T_{N}^{\prime}(z)\right|^{2} d A(z) & \leq(1+o(1)) \int_{L}\left|P_{m}(z)\right|^{2} d \mu(z) \\
& =(1+o(1)) \lambda_{m}\left(\mu, z_{0}\right),
\end{array}
$$

which follows precisely as (5.11). On the set $L_{\rho}^{*} \backslash U$ we use (5.25) to conclude that the integral

is

$$
\frac{W\left(z_{0}\right)}{\left|T_{N}^{\prime}\left(z_{0}\right)\right|^{2}} \int_{L_{\rho}^{*}}\left|P_{m}(z) S_{m}(z)\right|^{2}\left|T_{N}^{\prime}(z)\right|^{2} d A(z)
$$

$$
\begin{aligned}
& \leq(1+o(1)) \int_{L}\left|P_{m}(z)\right|^{2} d \mu(z)+C \exp \left(-m^{1 / 2}\right) \\
& =(1+o(1)) \lambda_{m}\left(\mu, z_{0}\right)+C \exp \left(-m^{1 / 2}\right) .
\end{aligned}
$$

Now if the right hand sides are $(1+o(1)) \lambda_{m}\left(\mu, z_{0}\right)$, then this is the complete analogue of (5.11), and from here the argument that led to (5.12) (and then to (5.16)) gives

$$
\begin{aligned}
& \liminf _{m \rightarrow \infty} m^{2} \lambda_{m}\left(\mu, z_{0}\right) \geq 2 \pi W\left(z_{0}\right) \frac{N^{2}}{\left|T_{N}^{\prime}\left(z_{0}\right)\right|^{2}}=2 \pi W\left(z_{0}\right)\left(\frac{\partial g_{\overline{\mathbf{C} \backslash L}}\left(z_{0}, \infty\right)}{\partial \mathbf{n}}\right)^{-2} \\
& \geq 2 \pi W\left(z_{0}\right)\left(\frac{\partial g_{\overline{\mathbf{C} \backslash K}}\left(z_{0}, \infty\right)}{\partial \mathbf{n}}+\varepsilon\right)^{-2},
\end{aligned}
$$

and upon $\varepsilon \rightarrow 0$ we get the desired lower estimate.

Thus, all we have to show is that

$$
\exp \left(-m^{1 / 2}\right)=o\left(\lambda_{m}\left(\mu, z_{0}\right)\right)
$$

which certainly follows, since

$$
\left.\lambda_{m}\left(\mu, z_{0}\right)\right) \geq \lambda_{m}\left(\left.W\right|_{U \cap K}, z_{0}\right) .
$$

Here the right hand side is $\geq c / m^{2}$ for some $c>0$, since we can apply Theorem 1.3 to the weight $W$ on the set $\overline{U \cap K}=\overline{\Delta_{\delta}\left(z_{0}\right)} \cap K$, the boundary of which consists of 
two $C^{2}$ arcs: one is a subarc of $\partial K$, and the other is a subarc of the boundary circle of $\overline{\Delta_{\delta}\left(z_{0}\right)}$ (note also that $W$ lies in between $W\left(z_{0}\right) / 2$ and $2 W\left(z_{0}\right)$ on $\bar{U} \cap K$ ).

\section{Proof of Theorems 1.1 and 1.2}

6.1. Lower estimate for arc length measure. In this section we consider Theorem 1.1 for $\mu=s_{\gamma}$, the arc measure on $\gamma$. In what follows we set $K=\operatorname{Pc}(\gamma)$ (this is the union of the closed domains that the different components of $\gamma$ enclose).

Let $P_{n}$ be the polynomial of degree at most $n$ that takes the value 1 at $z_{0} \in \gamma$ and has minimal $L^{2}\left(d s_{\gamma}\right)$-norm (equal to $\left.\lambda_{n}\left(d s_{\gamma}, z_{0}\right)^{1 / 2}\right)$. Consider the polynomials $S_{n}=S_{n, z_{0}, K}$ of degree at most $n^{109 / 110}$ from Corollary 4.2. We claim that $\left|P_{n} S_{n}\right|$ takes its maximum on $\gamma$ only in $\Delta_{n^{-9 / 10}}\left(z_{0}\right)$. Note that $P_{n}\left(z_{0}\right) S_{n}\left(z_{0}\right)=1$, so the maximum in question is at least 1 . If the claim is not true, then there is a $z^{*} \in \gamma \backslash \Delta_{n^{-9 / 10}}\left(z_{0}\right)$ with $\left|P_{n}\left(z^{*}\right) S_{n}\left(z^{*}\right)\right| \geq 1$, and then by the choice of $S_{n}$ we have

$$
\left|P_{n}\left(z^{*}\right)\right| \geq \exp \left(c_{0} n^{1 / 110}\right) / C_{0} \text {. }
$$

Let $z^{* *}$ be a point on $\gamma$ where $\left|P_{n}\right|$ takes its maximum $M \geq \exp \left(c_{0} n^{1 / 110}\right) / C_{0}$. By Corollary 7.2 from Section 7 we have $\left|P_{n}^{\prime}(z)\right| \leq C n^{2} M$ for $\operatorname{dist}(z, \gamma) \leq 1 / n^{2}$. Hence for $z \in \Delta_{1 / 2 C n^{2}}\left(z^{* *}\right), z \in \gamma$ we have (with integration along the straight segment)

$$
\left|P_{n}(z)\right| \geq\left|P_{n}\left(z^{* *}\right)\right|-\left|\int_{z^{* *}}^{z} P_{n}^{\prime}(t) d t\right| \geq M-\frac{C n^{2} M}{2 C n^{2}}=M / 2 .
$$

But $\Delta_{1 / 2 C n^{2}}\left(z^{* *}\right) \cap \gamma$ has $s_{\gamma}$ measure $\geq 1 / 2 C n^{2}$ (just consider that the center $z^{* *}$ of the disk $\Delta_{1 / 2 C n^{2}}\left(z^{* *}\right)$ lies on $\gamma$, and $\gamma$ has to reach the boundary of that disk as we follow it from $z^{* *}$ in either direction). Therefore,

$$
\begin{aligned}
\int_{\gamma}\left|P_{n}\right|^{2} d s_{\gamma} & \geq \int_{\gamma \cap \Delta_{1 / 2 C n^{2}\left(z^{* *}\right)}}\left|P_{n}\right|^{2} d s_{\gamma} \\
& \geq(M / 2)^{2} / 2 C n^{2} \geq \exp \left(2 c_{0} n^{1 / 110}\right) / 8 C C_{0}^{2} n^{2} \rightarrow \infty,
\end{aligned}
$$

which is impossible. This contradiction proves the claim.

Thus, if $z_{1}$ denotes a point on $\gamma$ where $\left|P_{n} S_{n}\right|$ takes its maximum, then $\mid z_{1}-$ $z_{0} \mid \leq n^{-9 / 10}$. Next we mention that $\partial g_{\overline{\mathbf{C}} \backslash \gamma} / \partial \mathbf{n}$ is continuous around $z_{0}$ by the last sentence in Theorem 3.2 . Therefore,

$$
\frac{\partial g_{\overline{\mathbf{C} \backslash \gamma}}\left(z_{1}, \infty\right)}{\partial \mathbf{n}}=(1+o(1)) \frac{\partial g_{\overline{\mathbf{C} \backslash \gamma}}\left(z_{0}, \infty\right)}{\partial \mathbf{n}} .
$$

For $\varepsilon>0$ let $\sigma=\sigma_{z_{1}}$ be a lemniscate inside $\gamma$ such that $\sigma$ touches $\gamma$ at $z_{1}$, and if $L$ is the closed lemniscate domain that $\sigma$ encloses, then

$$
\frac{\partial g_{\overline{\mathbf{C} \backslash L}}\left(z_{1}, \infty\right)}{\partial \mathbf{n}} \leq \frac{\partial g_{\overline{\mathbf{C} \backslash \gamma}}\left(z_{1}, \infty\right)}{\partial \mathbf{n}}+\varepsilon .
$$

The existence of $\sigma$ was stated in Theorem 2.3. where it was also shown (and this is absolutely crucial in the proof below) that for $z_{1}$ lying sufficiently close to $z_{0}$ this $\sigma_{z_{1}}$ can be chosen to be a translated and rotated copy of a fixed lemniscate, say of $\sigma_{z_{0}}$. Set

$$
R_{n}(z)=\frac{P_{n}(z) S_{n, z_{0}, K}(z) S_{n, z_{1}, K}(z)}{P_{n}\left(z_{1}\right) S_{n, z_{0}, K}\left(z_{1}\right)}
$$

with the polynomials $S_{n, Z, K}$ from Corollary 4.2, Then $\operatorname{deg}\left(R_{n}\right) \leq n+2 n^{109 / 110}$, $R_{n}\left(z_{1}\right)=1$ and $\left|R_{n}(z)\right| \leq\left|P_{n}(z)\right|$ on $\gamma$ (recall that the denominator in the definition of $R_{n}$ is at least 1 in absolute value, for $z_{1}$ was a maximum point for $\left.\left|P_{n} S_{n, z_{0}, K}\right|\right)$. 
Let $\sigma=\left\{z|| T_{N}(z) \mid=1\right\}$ with some polynomial $T_{N}$ of degree $N$, where $N$ depends only on $z_{0}$ and not on $z_{1}$ (which also depends on $n$ ). We may assume that $T_{N}\left(z_{1}\right)=1$. Then $T^{-1}(-1)$ cuts $\sigma$ into $N$ arcs $\sigma_{1}, \ldots, \sigma_{N}$ numbered so that $z_{1} \in \sigma_{1}$. Since $z_{1}$ was a maximum point for $\left|P_{n} S_{n, z_{0}, K}\right|$, it follows that

$$
\left|R_{n}(z)\right| \leq C_{0} e^{-c_{0} n^{1 / 110}}, \quad z \in \sigma_{2} \cup \cdots \cup \sigma_{N},
$$

$\left|R_{n}(z)\right| \leq 1$ on $\sigma_{1}$ and $\left|R_{n}(z)\right| \leq\left|P_{n}(z)\right|$ on $\gamma$. Now $\sigma_{z_{1}}$ and $\gamma$ are uniformly $C^{2}$ in a neighborhood of $z_{1}$ (recall that $\sigma_{z_{1}}$ is a rotated and translated copy of $\sigma_{z_{0}}$ ), and they touch each other at $z_{1}$. Therefore in their parametric representation with respect to arc length, corresponding points are quadratically close to each other around $z_{1}$ : if for a $t \in \gamma,\left|t-z_{1}\right| \leq 3 n^{-9 / 10}$ the point $t^{*} \in \sigma$ is determined by $s_{\sigma}\left(\left[z_{1}, t^{*}\right]\right)=s_{\gamma}\left(\left[z_{1}, t\right]\right)$ (actually, there are two such points; take the one lying closer to $t$, then the mapping $q(t)=t^{*}$ is $1-$ to -1 and $|q(t)-t| \leq C\left|t-z_{1}\right|^{2}$ with some constant $C$. By the definition of $t^{*}=q(t)$ we have $d s_{\sigma}\left(t^{*}\right)=d s_{\gamma}(t)$. Therefore, if $I_{n}$ denotes the arc of $\sigma$ consisting of the points $t^{*}$ with $\left|t^{*}-z_{1}\right| \leq n^{-9 / 10}$, then

$$
\int_{t^{*} \in I_{n}}\left|R_{n}\left(t^{*}\right)\right|^{2} d s_{\sigma}\left(t^{*}\right)=\int_{t^{*} \in I_{n}}\left|R_{n}(q(t))\right|^{2} d s_{\gamma}(t),
$$

and certainly $\left|t-z_{1}\right| \leq 2 n^{-9 / 10}$ if $\left|t^{*}-z_{1}\right| \leq n^{-9 / 10}$. Corollary 7.4 in Section 7 gives with some constant $D$ (recall that $\left\|R_{n}\right\|_{\gamma} \leq 1$ )

$\left|R_{n}(q(t))-R_{n}(t)\right| \leq D n \cdot|q(t)-t| \leq D n \cdot C\left|t-z_{1}\right|^{2} \leq 4 D C n \cdot n^{-18 / 10}=D_{1} n^{-4 / 5}$

for $\left|t^{*}-z_{1}\right| \leq n^{-9 / 10}$. Now

$$
\begin{aligned}
& \left.\left|\int_{t^{*} \in I_{n}}\right| R_{n}\left(t^{*}\right)\right|^{2} d s_{\sigma}\left(t^{*}\right)-\int_{t^{*} \in I_{n}}\left|R_{n}(t)\right|^{2} d s_{\gamma}(t) \mid \\
& \quad=\left|\int_{t^{*} \in I_{n}}\left(\left|R_{n}(q(t))\right|^{2}-\left|R_{n}(t)\right|^{2}\right) d s_{\gamma}(t)\right|,
\end{aligned}
$$

and if the right hand side is written symbolically as

$$
\left|\int\left(|A|^{2}-|B|^{2}\right)\right|
$$

then by Hölder's and Minkowski's inequalities we can continue this as

$$
\begin{aligned}
& \left|\int(|A|-|B|)(|A|+|B|)\right| \leq\left(\int|A-B|^{2}\right)^{1 / 2}\left\{\left(\int|A|^{2}\right)^{1 / 2}+\left(\int|B|^{2}\right)^{1 / 2}\right\} \\
& \leq D_{1} n^{-4 / 5}\left\{\left(\int|A|^{2}\right)^{1 / 2}+\left(\int|B|^{2}\right)^{1 / 2}\right\},
\end{aligned}
$$

where in the last step we used (6.6). But the integrands in the last two integrals on the right hand side are at most 1 , and the integration is done over a set of $s_{\gamma}$-measure $\leq D_{2} n^{-9 / 10}$ (recall that we are working inside $J$, which is a $C^{2}$ arc). Therefore the last two integrals are at most $D_{2}^{1 / 2} n^{-9 / 20}$, and hence

$$
\left.\left|\int_{t^{*} \in I_{n}}\right| R_{n}\left(t^{*}\right)\right|^{2} d s_{\sigma}\left(t^{*}\right)-\int_{t^{*} \in I_{n}}\left|R_{n}(t)\right|^{2} d s_{\gamma}(t) \mid \leq D_{3} n^{-4 / 5-9 / 20}=D_{3} n^{-5 / 4} .
$$


Note that here on the left hand side the first integral is on a part of $\sigma$, while the second integral is on the corresponding part of $\gamma$. In any case

$$
\int_{t^{*} \in I_{n}}\left|R_{n}^{2}\left(t^{*}\right)\right|^{2} d s_{\sigma}\left(t^{*}\right) \leq \int_{\gamma}\left|R_{n}^{2}(t)\right|^{2} d s_{\gamma}(t)+D_{3} n^{-5 / 4} \leq \lambda_{n}\left(s_{\gamma}, z_{0}\right)+D_{3} n^{-5 / 4} .
$$

For $\left|t^{*}-z_{0}\right| \geq n^{-9 / 10}$ we have, by the definition of $R_{n}$ and of $S_{n, z_{1}, K}$ (recall that $z_{1}$ was a maximum point for $\left.\left|P_{n} S_{n, z_{0}, K}\right|\right)$, the inequality

$$
\left|R_{n}\left(t^{*}\right)\right| \leq C_{0} e^{-c_{0} n^{1 / 110}}, \quad\left|t^{*}-z_{0}\right| \geq 10^{-9 / 10},
$$

and we obtain from (6.8)

$$
\int_{\sigma}\left|R_{n}\left(t^{*}\right)\right|^{2} d s_{\sigma}\left(t^{*}\right) \leq \lambda_{n}\left(s_{\gamma}, z_{0}\right)+D_{4} n^{-5 / 4} .
$$

The same proof gives

$$
\int_{\sigma}\left|R_{n}\left(t^{*}\right)\right|^{2}\left|T_{N}^{\prime}\left(t^{*}\right)\right| d s_{\sigma}\left(t^{*}\right) \leq(1+o(1))\left|T_{N}^{\prime}\left(z_{0}\right)\right| \lambda_{n}\left(s_{\gamma}, z_{0}\right)+D_{5} n^{-5 / 4}
$$

because

$$
\left|R_{n}\left(t^{*}\right)\right|^{2}\left|T_{N}^{\prime}\left(t^{*}\right)\right|=(1+o(1))\left|T_{N}^{\prime}\left(z_{0}\right)\right|\left|R_{n}\left(t^{*}\right)\right|^{2}
$$

for $\left|t-z_{1}\right| \leq 2 n^{-9 / 10}$, and the left hand side is very small otherwise (see (6.9)).

For a $t^{*} \in \sigma$ recall the points $t_{j}^{*}$ from Section 5 defined as $T_{N}\left(t_{j}^{*}\right)=T_{N}(t)$, and as in Section 2 we have

$$
\sum_{j=1}^{N} R_{n}\left(t_{j}^{*}\right)=Q_{n}\left(T_{N}\left(t^{*}\right)\right)
$$

with some polynomial $Q_{n}$ of degree at most $\operatorname{deg}\left(R_{n}\right) / N \leq\left(n+2 n^{9 / 10}\right) / N$. Because of (6.5), in the defining sum of $Q_{n}\left(T_{N}\left(t^{*}\right)\right)$ all but one term is very small; hence

$$
\left|Q_{n}\left(T_{N}\left(t^{*}\right)\right)\right|^{2}=\left|\sum_{j=1}^{N} R_{n}\left(t_{j}^{*}\right)\right|^{2}=\sum_{j=1}^{N}\left|R_{n}\left(t_{j}^{*}\right)\right|^{2}+C_{0} N^{2} e^{-c_{0} n^{1 / 110}} .
$$

We get from (2.12)

$$
\int_{\sigma}\left(\sum_{i=1}^{N}\left|R_{n}\left(t_{i}^{*}\right)\right|^{2}\right)\left|T_{N}^{\prime}\left(t^{*}\right)\right| d s_{\sigma}\left(t^{*}\right)=N \int_{\sigma}\left|R_{n}\left(t^{*}\right)\right|^{2}\left|T_{N}^{\prime}\left(t^{*}\right)\right| d s_{\sigma}\left(t^{*}\right) .
$$

From here and from (6.10) - 6.11) it follows that the integral

$$
\int_{\sigma}\left|Q_{n}\left(T_{N}\left(t^{*}\right)\right)\right|^{2}\left|T_{N}^{\prime}\left(t^{*}\right)\right| d s_{\sigma}\left(t^{*}\right)
$$

is at most

$$
(1+o(1)) N\left|T_{N}^{\prime}\left(z_{0}\right)\right| \lambda_{n}\left(s_{\gamma}, z_{0}\right)+D_{5} N^{3} n^{-5 / 4} .
$$

By (2.14) the integral in (6.13) is nothing other than

$$
N \int_{\mathcal{C}}\left|Q_{n}(\theta)\right|^{2} d s_{\mathcal{C}}(\theta)
$$

where $\mathcal{C}$ denotes the unit circle, and so this is less than (6.14). Since

$$
Q_{n}(1)=Q_{n}\left(T_{N}\left(z_{1}\right)\right)=\sum_{j=1}^{N} R_{n}\left(\left(z_{1}\right)_{j}\right)=1+o(1),
$$


this immediately implies with $m=\operatorname{deg}\left(Q_{n}\right)$ the inequality

$$
\lambda_{m}\left(s_{\mathcal{C}}, 1\right) \leq(1+o(1))\left|T_{N}^{\prime}\left(z_{0}\right)\right| \lambda_{n}\left(s_{\gamma}, z_{0}\right)+D_{5} N^{2} n^{-5 / 4} .
$$

To complete the proof of the lower estimate we need to compute the left hand side. This will be done in (6.19) below, and from it and from the previous formula we obtain

$$
\frac{2 \pi}{m+1} \leq(1+o(1))\left|T_{N}^{\prime}\left(z_{0}\right)\right| \lambda_{n}\left(s_{\gamma}, z_{0}\right)+D_{5} N^{2} n^{-5 / 4}
$$

Now since $m \leq\left(n+2 n^{9 / 10}\right) / N$, the estimate

$$
(1+o(1)) \frac{2 \pi N}{n\left|T_{N}^{\prime}\left(z_{0}\right)\right|}-D_{6} N^{2} n^{-5 / 4} \leq \lambda_{n}\left(s_{\gamma}, z_{0}\right)
$$

follows. Hence

$$
\liminf _{n \rightarrow \infty} n \lambda_{n}\left(s_{\gamma}, z_{0}\right) \geq \frac{2 \pi N}{\left|T_{N}^{\prime}\left(z_{0}\right)\right|}=2 \pi\left(\frac{\partial g_{\overline{\mathbf{C} \backslash L}}\left(z_{0}, \infty\right)}{\partial \mathbf{n}}\right)^{-1}
$$

where we used (2.2). Finally, (6.4) shows, upon letting $\varepsilon \rightarrow 0$,

$$
\liminf _{n \rightarrow \infty} n \lambda_{n}\left(s_{\gamma}, z_{0}\right) \geq 2 \pi\left(\frac{\partial g_{\overline{\mathbf{C}} \backslash \gamma}\left(z_{0}, \infty\right)}{\partial \mathbf{n}}\right)^{-1}
$$

In this proof we have used (6.19) below, which we now verify. Let $\tilde{Q}_{m}(\theta)=$ $\sum_{j=1}^{m} a_{j} \theta^{j}$ be a polynomial of degree at most $m$ with $Q_{m}(1)=1$. Then $\sum_{j=1}^{m} a_{j}=1$ and

$$
\int_{\mathcal{C}}\left|\tilde{Q}_{m}(\theta)\right|^{2} d s_{\mathcal{C}}(\theta)=\int_{0}^{2 \pi}\left|\tilde{Q}_{m}\left(e^{i t}\right)\right|^{2} d t=2 \pi \sum_{j=0}^{m}\left|a_{j}\right|^{2}
$$

By the Cauchy-Schwarz inequality

$$
1=\sum_{j=0}^{m} a_{j} \leq\left(\sum_{j=0}^{m}\left|a_{j}\right|^{2}\right)^{1 / 2}((m+1))^{1 / 2}
$$

i.e.

$$
\sum_{j=0}^{m}\left|a_{j}\right|^{2} \geq \frac{1}{m+1}
$$

and we have equality if $a_{j}=1 /(m+1)$, i.e. if

$$
\tilde{Q}_{m}(\theta)=\frac{1}{m+1} \sum_{j=0}^{m} \theta^{j}
$$

This verifies

$$
\lambda_{m}\left(s_{\mathcal{C}}, 1\right)=\frac{2 \pi}{m+1},
$$

and the proof of the lower estimate is complete. 
6.2. Proof of Theorem 1.2, the lower estimate for all measures. Let $J$ be the arc from the theorem, $z_{0} \in J$. The assumption is that in a neighborhood of $z_{0}$ the measure $\mu$ is of the form $d \mu(t)=w(t) d s_{\gamma}(t)$ with some $w$ that is continuous at $z_{0}$. We may assume that this representation holds true on $J$. Since we want to prove a lower estimate in this subsection, we may assume that $w\left(z_{0}\right)>0$ (otherwise the lower estimate is the trivial 0 ).

Let $P_{n}$ be a polynomial of degree at most $n$ such that $P_{n}\left(z_{0}\right)=1$ and $\int\left|P_{n}\right|^{2} d \mu=$ $\lambda_{n}\left(\mu, z_{0}\right)$. We shall closely follow the proof from the previous subsection, and for that purpose we are going to denote $J$ by $\gamma$.

For a given $\varepsilon>0$ let $\sigma$ be a lemniscate inside $\operatorname{Pc}(K)$ such that $\sigma$ touches $J=\gamma$ at $z_{0}$, and for the closed lemniscate domain $L=L_{\sigma}$ that it encloses, we have

$$
\frac{\partial g_{\overline{\mathbf{C}} \backslash L}\left(z_{0}, \infty\right)}{\partial \mathbf{n}} \leq \frac{\partial g_{\overline{\mathbf{C}} \backslash K}\left(z_{0}, \infty\right)}{\partial \mathbf{n}}+\varepsilon .
$$

Since $\operatorname{cap}(K)=\operatorname{cap}(\operatorname{Int}(\operatorname{Pc}(K)))$, the existence of $\sigma$ follows exactly as in subsection 5.3 (in that proof set $W \equiv 1$ on $\operatorname{Pc}(K)$ ).

The previous proof in subsection 6.1 was based on the fact that

$$
\left|P_{n} S_{n}\right| \text { takes its maximum on } K \text { only in } \Delta_{n^{-9 / 10}}\left(z_{0}\right) \text {. }
$$

First we show that in the general case (6.21) is enough to deduce the proper lower estimate for $\lambda_{n}\left(\mu, z_{0}\right)$. We follow the proof from subsection 6.1. In fact, (6.21) leads to (6.7), which implies

$$
\begin{aligned}
\int_{t^{*} \in I_{n}}\left|R_{n}\left(t^{*}\right)\right|^{2} d s_{\sigma}\left(t^{*}\right) & \leq \int_{t^{*} \in I_{n}}\left|R_{n}(t)\right|^{2} d s_{\gamma}(t)+D_{3} n^{-5 / 4} \\
& \leq \int_{t^{*} \in I_{n}}\left|P_{n}(t)\right|^{2} d s_{\gamma}(t)+D_{3} n^{-5 / 4}
\end{aligned}
$$

and we used this in the form (6.8). Now the weight $w$ (certainly defined on $\gamma \cap$ $\left.\Delta_{3 n^{-9 / 10}}\left(z_{0}\right)\right)$ is positive and continuous at $z_{0}$, so (6.22) implies

$$
\begin{aligned}
& \int_{t^{*} \in I_{n}}\left|R_{n}\left(t^{*}\right)\right|^{2}\left|T_{N}^{\prime}\left(t^{*}\right)\right| d s_{\sigma}\left(t^{*}\right) \\
& \quad \leq(1+o(1)) \frac{\left|T_{N}^{\prime}\left(z_{0}\right)\right|}{w\left(z_{0}\right)} \int_{t^{*} \in I_{n}}\left|P_{n}(t)\right|^{2} w(t) d s_{\gamma}(t)+D_{3} n^{-5 / 4}
\end{aligned}
$$

with a possibly larger constant $D_{3}$ than previously noted. This, combined with (6.9), yields

$$
\begin{aligned}
& \int_{t^{*} \in \sigma}\left|R_{n}\left(t^{*}\right)\right|^{2}\left|T_{N}^{\prime}\left(t^{*}\right)\right| d s_{\sigma}\left(t^{*}\right) \leq(1+o(1)) \frac{\left|T_{N}^{\prime}\left(z_{0}\right)\right|}{w\left(z_{0}\right)} \int_{t^{*} \in I_{n}}\left|P_{n}(t)\right|^{2} w(t) d s_{\gamma}(t) \\
& +D_{3} n^{-5 / 4}
\end{aligned}
$$

and hence

$$
\begin{aligned}
\int_{t^{*} \in \sigma}\left|R_{n}\left(t^{*}\right)\right|^{2}\left|T_{N}^{\prime}\left(t^{*}\right)\right| d s_{\sigma}\left(t^{*}\right) \leq & (1+o(1)) \frac{\left|T_{N}^{\prime}\left(z_{0}\right)\right|}{w\left(z_{0}\right)} \int_{\gamma}\left|P_{n}(t)\right|^{2} d \mu(t) \\
& +D_{3} n^{-5 / 4} \\
= & (1+o(1)) \frac{\left|T_{N}^{\prime}\left(z_{0}\right)\right|}{w\left(z_{0}\right)} \lambda_{n}\left(\mu, z_{0}\right)+D_{3} n^{-5 / 4} .
\end{aligned}
$$


From here the rest of the proof in the previous subsection leads to (see (6.10) (6.16) )

$$
\liminf _{n \rightarrow \infty} n \lambda_{n}\left(\mu, z_{0}\right) \geq w\left(z_{0}\right) \frac{2 \pi N}{\left|T_{N}^{\prime}\left(z_{0}\right)\right|}=2 \pi w\left(z_{0}\right)\left(\frac{\partial g_{\overline{\mathbf{C} \backslash L}}\left(z_{0}, \infty\right)}{\partial \mathbf{n}}\right)^{-1},
$$

and finally to (see (6.17) and (6.201)

$$
\liminf _{n \rightarrow \infty} n \lambda_{n}\left(\mu, z_{0}\right) \geq 2 \pi w\left(z_{0}\right)\left(\frac{\partial g_{\overline{\mathbf{C} \backslash K}}\left(z_{0}, \infty\right)}{\partial \mathbf{n}}\right)^{-1} .
$$

This is precisely the lower estimate that gives one half of Theorem 1.2 ,

Let $H_{n}$ be a polynomial of degree $o(n)$ such that $H_{n}\left(z_{0}\right)=1$ and $\left|H_{n}(z)\right| \leq 1$ for $z \in K$. Then in the proof we have just given, $P_{n}$ (and then $R_{n}$ ) can be replaced by $P_{n} H_{n}\left(P_{n} H_{n} S_{n}\right)$ without affecting the proof. Thus, if we can show that for some $H_{n}$

$$
\left|P_{n} H_{n} S_{n}\right| \text { takes its maximum on } K \text { only in } \Delta_{n^{-9 / 10}}\left(z_{0}\right) \text {, }
$$

then the proof goes through word for word.

In subsection 6.1 the polynomial $P_{n}$ for which (6.21) was proved was a minimal polynomial for $\lambda_{n}\left(s_{\gamma}, z_{0}\right)$, but now our $P_{n}$ is a minimal polynomial for $\lambda_{n}\left(\mu, z_{0}\right)$. The proof of (6.21) in subsection 6.1 was based on two facts (see the beginning of the proof in subsection 6.1):

1) Markov's inequality (Theorem 7.1).

2) The $s_{\gamma}$-measure of $\Delta_{1 / 2 C n^{2}}(Z) \cap \gamma$ is at least $1 / 2 C n^{2}$ for $Z \in \gamma$ (there we had $\left.Z=z^{* *}\right)$.

Of these two, Markov's inequality is general and does not depend on $\mu$, so it is certainly at our disposal. However, the analogue of 2 ) is not true for some measures $\mu$, for the $\mu$-measure of $\Delta_{1 / 2 C n^{2}}(Z) \cap \gamma$ can be much smaller than $1 / n^{2}$. Note that this cannot happen when $Z$ lies close to $z_{0}$, for $\mu$ is absolutely continuous with respect to $s_{\gamma}$ around $z_{0}$ and it has positive and continuous derivative $d \mu / d s_{\gamma}$ there. Therefore, we shall select $H_{n}$ so that $P_{n} H_{n}$ is small on $\gamma$ outside a given neighborhood of $z_{0}$; the rest of the proof remains the same.

To achieve this we repeat the argument leading to (5.25): $\mu$ being in the $\mathbf{R e g}$ class implies (see [33, Thorem 3.2.3]) that

$$
\limsup _{n \rightarrow \infty} \frac{\left\|Q_{n}\right\|_{\gamma}^{1 / n}}{\left\|Q_{n}\right\|_{L^{2}(\mu)}^{1 / n}} \leq 1
$$

In particular, for $P_{n}$ (the extremal polynomials for $\lambda_{n}\left(\mu, z_{0}\right)$ ) we get the relation

$$
\left\|P_{n}\right\|_{\gamma} \leq e^{\varepsilon_{n} n}
$$

with some $\varepsilon_{n} \rightarrow 0$. We may assume $\varepsilon_{n} \geq 1 / n^{1 / 2}$. Now let $U=\Delta_{\delta}\left(z_{0}\right)$ be a neighborhood of $z_{0}$ such that $w>w\left(z_{0}\right) / 2>0$ on $\Delta_{2 \delta}\left(z_{0}\right) \cap \gamma$. Choose $\beta=3 / 2$, $Z=z_{0}$ in Theorem 4.1. consider the polynomials in that theorem of degree $n \varepsilon_{n}^{1 / 2}$, and denote them by $H_{n}$; i.e. $H_{n}$ is of degree at most $n \varepsilon_{n}^{1 / 2}, H_{n}\left(z_{0}\right)=1,\left|H_{n}(z)\right| \leq 1$ for $z \in K$ and

$$
\left|H_{n}(z)\right| \leq D_{\beta} e^{-\left(n \varepsilon_{n}^{1 / 2}\right) d_{\beta}\left|z-z_{0}\right|^{\beta}} .
$$


Then for $z \notin U, z \in \gamma$ we have (see (6.30) )

$$
\begin{aligned}
\left|P_{n}(z) H_{n}(z)\right| & \leq D_{\beta} \exp \left(\varepsilon_{n} n-n \varepsilon_{n}^{1 / 2} d_{\beta}\left|z-z_{0}\right|^{\beta}\right) \\
& \leq D_{\beta} \exp \left(\varepsilon_{n} n-n \varepsilon_{n}^{1 / 2} d_{\beta} \delta^{\beta}\right)<1 / 2<P_{n}\left(z_{0}\right) H_{n}\left(z_{0}\right)
\end{aligned}
$$

for all large $n$. Hence, if $z_{1}$ is a maximum point for $\left|P_{n} H_{n} S_{n, z_{0}, K}\right|$ on $K$, then necessarily $z_{1} \in U$, and then the proof in between (6.1) and (6.2) from subsection 6.1 can be applied: use Markov's inequality (see $\mathbf{1}$ ) above) and then note that since $z^{* *}$ must belong to $U$, the $\mu$-measure of the set $\Delta_{1 / 2 C n^{2}}(Z) \cap \gamma$ is at least $w\left(z_{0}\right) / 2$ times its $s_{\gamma}$-measure, i.e. it is at least $w\left(z_{0}\right) / 4 C n^{2}$. This way we get the statement (6.28), and, as we have already seen, it is enough to prove (6.27).

6.3. Proof of Theorem 1.2, upper estimate. We can be brief, for the argument should be familiar by now.

Recall that in a neighborhood of $z_{0}$ the set $K=\operatorname{supp}(\mu)$ is a $C^{2}$ arc $J$ and $d \mu(t)=w(t) d s_{J}(t)$ there, and, as in the previous subsection, we set $\gamma=J$.

Now let $\sigma=T_{N}^{-1}(\mathcal{C})$ be a lemniscate such that it touches $\gamma$ at $z_{0}$, the closed domain $L=T_{N}^{-1}(\bar{\Delta})$ it encloses contains $K$ in its interior (except for the point $z_{0}$ which must be on the boundary of $L$ ) and (for a given $\varepsilon>0$ )

$$
\frac{\partial g_{\overline{\mathbf{C}} \backslash L}\left(z_{0}, \infty\right)}{\partial \mathbf{n}} \geq \frac{\partial g_{\overline{\mathbf{C}} \backslash K}\left(z_{0}, \infty\right)}{\partial \mathbf{n}}-\varepsilon
$$

(see Theorem 2.3). We may assume $T_{N}\left(z_{0}\right)=1$. Let

$$
R_{n}(z)=\tilde{Q}_{n}\left(T_{N}(z)\right) S_{n, z_{0}, L}(z)
$$

with the $\tilde{Q}_{n}$ from (6.18) and the $S_{n, z_{0}, L}$ from Corollary 4.2. Then $R_{n}$ has degree at most $n N+n^{109 / 110}$. Exactly as before, the set $T_{N}^{-1}(-1)$ divides $\sigma$ into the subarcs $\sigma_{1}, \ldots, \sigma_{N}, z_{0} \in \sigma_{1}$. From (2.13) it follows that

$$
\begin{aligned}
\int_{\sigma_{1}}\left|R_{n}\left(t^{*}\right)\right|^{2}\left|T_{N}^{\prime}\left(t^{*}\right)\right| d s_{\sigma}\left(t^{*}\right) & \leq \int_{\sigma_{1}}\left|\tilde{Q}_{n}\left(T_{n}\left(t^{*}\right)\right)\right|^{2}\left|T_{N}^{\prime}\left(t^{*}\right)\right| d s_{\sigma}\left(t^{*}\right) \\
& =\int_{\mathcal{C}}\left|\tilde{Q}_{n}\right|^{2} d s_{\mathcal{C}}=\frac{2 \pi}{n+1} .
\end{aligned}
$$

Now $\sigma$ and $\gamma=J$ touch each other at $z_{0}$, so the reasoning that leads to (6.7) remains valid, and we get from (6.7) that

$$
\begin{aligned}
\int_{t^{*} \in I_{n}, t \in \gamma}\left|R_{n}(t)\right|^{2}\left|T_{N}^{\prime}(t)\right| d s_{\gamma}(t) \leq & \int_{t^{*} \in I_{n}, t^{*} \in \sigma}\left|R_{n}\left(t^{*}\right)\right|^{2}\left|T_{N}^{\prime}\left(t^{*}\right)\right| d s_{\sigma}\left(t^{*}\right) \\
& +D_{3} n^{-5 / 4} \leq \frac{2 \pi}{n+1}+D_{3} n^{-5 / 4} .
\end{aligned}
$$

At the same time, since $\left|\tilde{Q}_{n}\right|$ takes its maximum on the unit circle at 1, we get from the definition of $S_{n}$ that

$$
\left|R_{n}(t)\right| \leq C_{0} e^{-c_{0} n^{1 / 110}}, \quad\left|t-z_{0}\right| \geq n^{-9 / 10}, t \in \gamma .
$$

Now these last two formulae give

$$
\int_{\left|t-z_{0}\right| \geq n^{-9 / 10}}\left|R_{n}(t)\right|^{2} d \mu(t)+\int_{\left|t-z_{0}\right| \leq n^{-9 / 10}}\left|R_{n}(t)\right|^{2}\left|T_{N}^{\prime}(t)\right| d s_{\gamma}(t) \leq(1+o(1)) \frac{2 \pi}{n} .
$$


The same proof shows that if $H$ is continuous at $z_{0}$ and integrable with respect to $s_{\gamma}$, then

$$
\begin{aligned}
\int_{\left|t-z_{0}\right| \geq n^{-9 / 10}}\left|R_{n}(t)\right|^{2} d \mu(t) & +\int_{\left|t-z_{0}\right| \leq n^{-9 / 10}}\left|R_{n}(t)\right|^{2}\left|T_{N}^{\prime}(t)\right| H(t) d s_{\gamma}(t) \\
& \leq(1+o(1)) H\left(z_{0}\right) \frac{2 \pi}{n}
\end{aligned}
$$

and finally with $H(t)=w(t) /\left|T_{N}^{\prime}(t)\right|$ we get

$$
\begin{aligned}
\int_{\left|t-z_{0}\right| \geq n^{-9 / 10}}\left|R_{n}(t)\right|^{2} d \mu(t) & +\int_{\left|t-z_{0}\right| \leq n^{-9 / 10}}\left|R_{n}(t)\right|^{2} w(t) d s_{\gamma}(t) \\
& \leq(1+o(1)) w\left(z_{0}\right) \frac{2 \pi}{n\left|T_{N}^{\prime}\left(z_{0}\right)\right|} .
\end{aligned}
$$

For $m=\operatorname{deg}\left(R_{n}\right) \leq n N+n^{109 / 110}$ this gives

$$
\lambda_{m}\left(\mu, z_{0}\right) \leq(1+o(1)) 2 \pi w\left(z_{0}\right) \frac{N}{\left|T_{N}^{\prime}\left(z_{0}\right)\right|} \frac{1}{m},
$$

and in view of the monotonicity of $\lambda_{m}$ in $m$ this remains true for all $m$. This proves (see (2.2)) that

$$
\limsup _{m \rightarrow \infty} m \lambda_{m}\left(\mu, z_{0}\right) \leq 2 \pi w\left(z_{0}\right) \frac{N}{\left|T_{N}^{\prime}\left(z_{0}\right)\right|}=2 \pi w\left(z_{0}\right)\left(\frac{\partial g_{\overline{\mathbf{C}} \backslash L}\left(z_{0}, \infty\right)}{\partial \mathbf{n}}\right)^{-1},
$$

and finally, in view of (6.31) we obtain

$$
\limsup _{m \rightarrow \infty} m \lambda_{m}\left(\mu, z_{0}\right) \leq 2 \pi w\left(z_{0}\right)\left(\frac{\partial g_{\overline{\mathbf{C}} \backslash K}\left(z_{0}, \infty\right)}{\partial \mathbf{n}}\right)^{-1} .
$$

6.4. Proof of Theorem 1.1. In view of Theorem 1.2 it is enough to show that the condition " $d \mu(t) / d s_{\gamma}>0 s_{\gamma}$-almost everywhere" implies that the measure $\mu$ is in the Reg class (1.4). Let $\gamma_{1}, \ldots, \gamma_{k}$ be the components of $\gamma$ (each of them is a Jordan curve). By the localization theorem [33, Theorem 5.3.3] the regularity of $\mu$ is equivalent to the regularity of each $\left.\mu\right|_{\gamma_{j}}$. Hence we may assume that $\gamma$ consists of a single component, i.e. it is a rectifiable Jordan curve with outer domain $\Omega$.

Let $\omega_{\gamma}$ denote the equilibrium measure of $\gamma$. Theorem [33, Theorem 4.1.1] states that $\mu$ is regular if $d \mu(t) / d \omega_{\gamma}(t)>0 \omega_{\gamma}$-almost everywhere, so it is enough to show that $s_{\gamma}$ and $\omega_{\gamma}$ are mutually absolutely continuous with respect to each other. Let $\Phi: \Omega \rightarrow \overline{\mathbf{C}} \backslash \bar{\Delta}$ be the conformal map from the exterior of $\gamma$ onto the exterior of the unit disk. Then (see Theorem 3.1) $\Phi$ extends to a homeomorphism in between $\gamma$ and the unit circle $\mathcal{C}$, and by the theorem of F. and M. Riesz and I.I. Privalov (see [27, [0, II, Theorem VII,(10.17)] or [24, Theorem 6.8]) the rectifiability of $\gamma$ implies that $\Phi$ maps zero arc-measure sets into zero arc-measure sets and vice versa, i.e. a Borel set $E \subset \gamma$ has zero $s_{\gamma}$-measure if and only if $\Phi(\gamma)$ has zero Lebesgue measure (on the unit circle). On the other hand, $\omega_{\gamma}$ is the pull-back measure of the normalized arc measure on $\mathcal{C}: \omega_{\gamma}(E)=|\Phi(E)| / 2 \pi$, where $|\cdot|$ indicates arc length on $\mathcal{C}$ (see Theorem 3.1). Thus, again, $E$ has zero $\omega_{\gamma}$-measure if and only if $\Phi(E)$ has zero Lebesgue measure (on the unit circle). These prove the mutual absolute continuity of $s_{\gamma}$ and $\omega_{\gamma}$, and with this we have verified the regularity of $\mu$. 


\section{Markov and Bernstein type inequalities}

The following theorem is probably well known, but we have not found an exact reference, so for completeness we provide a proof. It has been an essential part of the proof of Theorem 1.2. Recall that a set on the plane is called a continuum if it is compact and connected and consists of more than one point.

Theorem 7.1. Let $K$ be a continuum. Then the Green's function $g_{\overline{\mathbf{C}} \backslash K}(z, \infty)$ is uniformly Hölder $1 / 2$ continuous on $K$, i.e. if $z_{0} \in \Omega$, then

$$
g_{\overline{\mathbf{C}} \backslash K}\left(z_{0}, \infty\right) \leq C \operatorname{dist}\left(z_{0}, K\right)^{1 / 2} .
$$

Here $C$ can be chosen to depend only on the diameter of $K$.

Proof. In this proof let $\mathcal{C}_{r}=\{|z|=r\}$ and $\Delta_{r}=\{|z|<r\}$, and denote by $\omega(z ; J, G)$ the harmonic measure of $J \subset \partial G$ at $z$ with respect to a domain $G$. Let $d$ be the diameter of $K$, and suppose that $0 \in K$. Then $K$ lies in $\overline{\Delta_{d}}$, and its logarithmic capacity is at least $d / 4$ (see [26, Theorem 5.3.2,a]). Hence formula (3.2) shows that

$$
g_{\overline{\mathbf{C} \backslash K}}(z, \infty) \leq \log 4 d+\log \frac{4}{d}=\log 16, \quad|z| \leq 3 d .
$$

Without loss of generality we may assume in proving (7.1) that the closest point in $K$ to $z$ is the origin. Then, by (7.2),

$$
g_{\overline{\mathbf{C}} \backslash K}(z, \infty) \leq(\log 16) \omega\left(z, \mathcal{C}_{3 d}, \Delta_{3 d} \backslash K\right),
$$

and by the Beurling-Nevanlinna theorem about the solution of Milloux's problem (see 22])

$$
(\log 16) \omega\left(z, \mathcal{C}_{3 d}, \Delta_{3 d} \backslash K\right) \leq(\log 16) \omega\left(|z|, \mathcal{C}_{3 d}, \Delta_{3 d} \backslash[-d, 0]\right) .
$$

On the other hand, $\operatorname{cap}(K) \leq \operatorname{cap}\left(\overline{\Delta_{d}}\right)=d$; hence (3.2) shows that

$$
g_{\overline{\mathbf{C}} \backslash[-d, 0]}(z, \infty) \geq \log 2 d-\log d=\log 2, \quad|z|=3 d .
$$

Therefore,

$$
\begin{aligned}
\omega\left(|z|, \mathcal{C}_{3 d}, \Delta_{3 d} \backslash[-d, 0]\right) & \leq \frac{1}{\log 2} g_{\overline{\mathbf{C} \backslash[-d, 0]}}(|z|, \infty) \\
& =\frac{1}{\log 2} \log \left|\sqrt{(2|z| / d+1)^{2}-1}+(2|z| / d+1)\right| \\
& \leq C_{d} \sqrt{|z|}
\end{aligned}
$$

where we used the fact that

$$
g_{\overline{\mathbf{C} \backslash[-1,1]}}(w, \infty)=\log \left|\sqrt{w^{2}-1}+w\right|
$$

and that the Green's function of $\overline{\mathbf{C}} \backslash[-d, 0]$ is obtained from this via the substitution $w=2 z / d+1$.

Now the theorem follows from the inequalities above.

Corollary 7.2. With the assumptions of Theorem 7.1, for every $D$ there is a constant $C_{D}$ such that if $P_{n}$ is a polynomial of degree at most $n$, then

$$
\left|P_{n}^{\prime}(z)\right| \leq C_{D} n^{2}\left\|P_{n}\right\|_{K}, \quad \operatorname{dist}(z, K) \leq D / n^{2} .
$$

A more precise estimate for $P_{n}^{\prime}$ but only on $K$ is in [24]. 
Proof. By the Bernstein-Walsh lemma ([39, p. 77] or [26, Theorem 5.5.7])

$$
\left|P_{n}(w)\right| \leq \exp \left(n g_{\overline{\mathbf{C} \backslash K}}(w, \infty)\right)\left\|P_{n}\right\|_{K} ;
$$

hence (7.1) gives for $\operatorname{dist}(w, K) \leq(D+1) / n^{2}$ the inequality

$$
\left|P_{n}(w)\right| \leq e^{C \sqrt{D+1}}\left\|P_{n}\right\|_{K} .
$$

Now if $\operatorname{dist}(z, K) \leq D / n^{2}$, then (7.3) follows if we apply Cauchy's formula for $P_{n}^{\prime}$ for $z$ and for the circle of radius $1 / n^{2}$ about $z$ :

$$
\left|P_{n}^{\prime}(z)\right|=\left|\frac{1}{2 \pi} \int_{|z-\xi|=n^{-2}} \frac{P_{n}(\xi)}{(\xi-z)^{2}} d \xi\right| \leq n^{2} \sup _{|z-\xi|=n^{-2}}\left|P_{n}(\xi)\right| \leq n^{2} e^{\sqrt{C(D+1)}}\left\|P_{n}\right\|_{K} .
$$

Theorem 7.3. Let $K$ be a compact set on the plane, $\Omega$ the unbounded component of $\overline{\mathbf{C}} \backslash K, J$ a closed $C^{2}$ arc on the boundary of $\Omega$ such that $J=\partial \Omega \cap O$ with some open set $O$, and let $J_{1}$ be a subarc of $J$ not having common endpoints with $J$. Then $g_{\overline{\mathbf{C}} \backslash K}(z, \infty)$ is uniformly Hölder 1 continuous on $J_{1}$, i.e. if $z_{0} \in \Omega$, then

$$
g_{\overline{\mathbf{C} \backslash K}}\left(z_{0}, \infty\right) \leq C \operatorname{dist}\left(z_{0}, J_{1}\right) .
$$

Proof. Clearly

$$
g_{\overline{\mathbf{C} \backslash K}}\left(z_{0}, \infty\right) \leq g_{\overline{\mathbf{C}} \backslash J}\left(z_{0}, \infty\right) .
$$

The conformal map $\Phi$ from $\overline{\mathbf{C}} \backslash J$ onto the exterior of the unit circle is $C^{1}$ on $J_{1}$, and

from which the statement follows.

$$
g_{\overline{\mathbf{C}} \backslash J}\left(z_{0}, \infty\right)=\log |\Phi(z)|,
$$

Corollary 7.4. With the assumptions and notation of Theorem 7.3 , for every $D$ there is a constant $C_{D}$ such that if $P_{n}$ is a polynomial of degree at most $n$, then

$$
\left|P_{n}^{\prime}(z)\right| \leq C_{D} n\left\|P_{n}\right\|_{K}, \quad \operatorname{dist}\left(z, J_{1}\right) \leq D / n .
$$

Proof. The proof is just a repetition of the proof of Corollary 7.2 if we use (7.5) instead of (7.1), and this time we integrate over the circle $|\xi-z|=1 / n$.

\section{REFERENCES}

1. T. Carleman, Über die Approximation analytischer Funktionen durch lineare Aggregate von vorgegebenen Potenzen, Ark. Mat. Astr. Fys., 17(1923), 215-244.

2. L. Carleson, Selected Problems on Exceptional Sets, Van Nostrand Mathematical Studies, 13, D. Van Nostrand Co., Inc., Princeton, N.J.-Toronto, Ont.-London, 1967. MR 0225986 $(37: 1576)$

3. W. Blaschke, Kreis und Kugel, Walter de Gruyter \& Co., Berlin, 1956. MR0077958 (17:1123d)

4. M. Findley, Universality for regular measures satisfying Szegö's condition locally, J. Approx. Theory, 155, 136-154.

5. G. Freud, Orthogonal Polynomials, Pergamon Press, Oxford, 1971.

6. L. Golinskii, The Christoffel function for orthogonal polynomials on a circular arc, J. Approx. Theory, 101(1999), 165-174. MR1726450(2001b:42032)

7. G. Golub, B. Gustafsson, P. Milanfar, M. Putinar and J. Varah, Shape reconstruction from moments: theory, algorithms and applications, SPIE Proceedings, Vol. 4116(2000), Advanced Signal Processing, Algorithms, Architecture and Implementations X (Franklin T. Luk, ed.), 406-416.

8. U. Grenander and G. Szegö, Toeplitz Forms and Their Applications, University of California Press, Berkeley and Los Angeles, 1958. MR0094840 (20:1349) 
9. B. Gustafsson, C. He, P. Milanfar and M. Putinar, Reconstructing planar domains from their moments, Inverse Problems, 16(2000), 1053-1070. MR1776483 (2001k:44010)

10. B. Gustafsson, M. Putinar, E. B. Saff and N. Stylianopoulos, Les polynômes orthogonaux de Bergman sur un archipel, C. R. Acad. Sci. Paris, Ser. I XXX (2008). MR2412785

11. B. Gustafsson, M. Putinar, E. B. Saff and N. Stylianopoulos, Bergman polynomials on an archipalego: estimates, zeros and shape reconstruction (manuscript, arXiv0811.1715v1).

12. K. G. Ivanov and V. Totik, Fast decreasing polynomials, Constructive Approximation, 6(1990), 1-20. MR.1027506 (90k:26023)

13. A. N. Kolmogorov, Stationary sequences in Hilbert spaces, Bull. Moscow State Univ., 2(1941), 1-40 (in Russian).

14. M. G. Krein, Generalization of investigations by G. Szegő, V. I. Smirnov and A. N. Kolmogorov, Dokl. Akad. Nauk. SSSR, 46(1945), 91-94 (in Russian). MR0013457 (7:156b)

15. A. L. Levin, E. B. Saff and N. Stylianopoulos, Zero distribution of Bergman orthogonal polynomials for certain planar domians, Constr. Approx., 19(2003), 411-435. MR1979059 (2004a:30007)

16. D. S. Lubinsky, A new approach to universality limits involving orthogonal polynomials, Annals of Mathematics (to appear).

17. A. Máté and P. Nevai, Bernstein's inequality in $L^{p}$ for $0<p<1$ and $(C, 1)$ bounds for orthogonal polynomials, Ann. of Math., 111(1980), 145-154. MR.558399 (81c:42003)

18. A. Máté, P. Nevai and V. Totik, Szegö's extremum problem on the unit circle, Annals of Math., 134(1991), 433-453. MR.1127481(92i:42014)

19. E. Miña-Díaz, E. B. Saff and N. S. Stylianopoulos, Zero distributions for polynomials orthogonal with weights over certain planar regions, Comput. Methods Funct. Theory, 5(2005), 185-221. MR2174353 (2006f:30004)

20. B. Nagy and V. Totik, Sharpening of Hilbert's lemniscate theorem, J. D'Analyse Math., 96(2005), 191-223. MR2177185 (2006g:30008)

21. P. Nevai, Géza Freud, Orthogonal polynomials and Christoffel functions. A case study, $J$. Approx. Theory, 48(1986), 1-167. MR862231(88b:42032)

22. R. Nevanlinna, Analytic Functions, Grundlehren der mathematischen Wissenschaften, 162, Springer-Verlag, Berlin, 1970. MR0279280(43:5003)

23. L. A. Pastur, Spectral and probabilistic aspects of matrix models. Algebraic and geometric methods in mathematical physics (Kaciveli, 1993), 207-242, Math. Phys. Stud., 19, Kluwer Acad. Publ., Dordrecht, 1996. MR1385683 (97b:82060)

24. Ch. Pommerenke, On the derivative of a polynomial, Michigan Math. J., 6(1959), 373-375. MR0109208 (22:95)

25. Ch. Pommerenke, Boundary Behavior of Conformal Mappings, Grundlehren der mathematischen Wissenschaften, 299, Springer-Verlag, Berlin, Heidelberg, New York, 1992. MR 1217706 (95b:30008)

26. T. Ransford, Potential Theory in the Complex Plane, Cambridge University Press, Cambridge, 1995 MR 1334766 (96e:31001)

27. F. Riesz and M. Riesz, Uber die Randwerte einer analytischen Funktion, Quatrieme Congrés de Math. Scand., 1916.

28. E. B. Saff and V. Totik, Logarithmic Potentials with External Fields, Grundlehren der mathematischen Wissenschaften, 316, Springer-Verlag, New York/Berlin, 1997. MR 1485778 (99h:31001)

29. B. Simon, Weak convergence of CD kernels and applications, Duke Math. J., 146(2009), no. 2, 305-330. MR2477763

30. B. Simon, The Christoffel-Darboux kernel, "Perspectives in PDE, Harmonic Analysis and Applications" in honor of V.G. Maz'ya's 70th birthday, to be published in Proceedings of Symposia in Pure Mathematics

31. B. Simon, Two extensions of Lubinsky's universality theorem, J. D'Analyse Math., 105(2008), 345-362 MR 2438429

32. B. Simon, Orthogonal Polynomials on the Unit Circle, V.1: Classical Theory, AMS Colloquium Series, American Mathematical Society, Providence, RI, 2005. MR2105088 (2006a:42002a)

33. H. Stahl and V. Totik, General Orthogonal Polynomials, Encyclopedia of Mathematics and its Applications, 43, Cambridge University Press, Cambridge, 1992. MR1163828 (93d:42029)

34. G. Szegö, Orthogonal Polynomials, Coll. Publ., XXIII, Amer. Math. Soc., Providence, 1975. 
35. G. Szegö, Collected Papers, ed. R. Askey, Birkhaüser, Boston-Basel-Stuttgart, 1982.

36. V. Totik, Asymptotics for Christoffel functions for general measures on the real line, $J$. D'Analyse Math., 81 (2000), 283-303. MR.1785285 (2001j:42021)

37. V. Totik, Polynomial inverse images and polynomial inequalities, Acta Math., 187 (2001), 139-160. MR:1864632 (2002h:41017)

38. V. Totik, Universality and fine zero spacing on general sets, Arkiv för Math., doi:10.1007/s11512-008-0071-3 (to appear)

39. J. L. Walsh, Interpolation and Approximation by Rational Functions in the Complex Domain, third edition, Amer. Math. Soc. Colloquium Publications, XX, Amer. Math. Soc., Providence, 1960. MR 0218587(36:1672a)

40. A. Zygmund, Trigonometric Series, Cambridge University Press, Cambridge, 1959. MR0107776 (21:6498)

Bolyai Institute, Analysis Research Group of the Hungarian Academy os Sciences, University of Szeged, Szeged, Aradi v. tere 1, 6720, Hungary - and - Department of Mathematics, University of South Florida, 4202 E. Fowler Avenue, PHy 114, Tampa, FLORIDA 33620-5700

E-mail address: totik@math.usf.edu 